\title{
Angular Energy Quantization for Linear Elliptic Systems with Antisymmetric Potentials and Applications
}

\author{
Paul Laurain \& Tristan Rivière
}

May 27, 2018

\begin{abstract}
In the present work we establish a quantization result for the angular part of the energy of solutions to elliptic linear systems of Schrödinger type with antisymmetric potentials in two dimension. This quantization is a consequence of uniform Lorentz-Wente type estimates in degenerating annuli. We derive from this angular quantization the full energy quantization for general critical points to functionals which are conformally invariant or also for pseudo-holomorphic curves on degenerating Riemann surfaces.
\end{abstract}

A.M.S. Classification : 35J47, 35J20, 35J60, 58E20, 53C42, 49Q05, 53C21, 32Q65

\section{Contents}

1 Lorentz spaces and standard Wente's inequalities 6

2 Wente type lemmas 7

3 Angular Energy Quantization for solutions to elliptic systems with anti-symmetric potential

4 Energy Quantization for critical points to conformally invariant lagrangians.

5 Other applications to pseudo-holomorphic curves, harmonic maps and Willmore surfaces

5.1 Lorentz-Wente type estimates for first order Wente type equations. . . . . . . . . . . . . .

5.2 Quantization of pseudo-holomorphic curves on degenerating Riemann surfaces . . . . . 28

5.3 Quantification for harmonic maps on a degenerating surfaces, a cohomoligical condition. 31

5.4 Energy Quantization for Willmore Surfaces. . . . . . . . . . . . . . . . . . . . 33

A Lorentz Estimates on Harmonic Functions.

\section{Introduction}

Conformal invariance is a fundamental property for many problems in physics and geometry. In the last decades it has become an important feature of many questions of non-linear analysis too. Elliptic conformally invariant lagrangians for instance share similar analysis behaviors : their Euler Lagrange equations are critical with respect to the function space naturally given by the lagrangian and, as a consequence, 
solutions to these Euler Lagrange equations are subject to concentration compactness phenomena. Questions such as the regularity of solutions or energy losses for sequences of solutions cannot be solved by robust general arguments in PDE but require instead a careful study of the interplay between the highest order part of the PDE and it's non-linearity.

For example, in dimension 2, let $(\Sigma, h)$ be a closed Riemann surface, it has been proved, see theorem I.2 of [Ri3], that every critical point of a conformally invariant functional, $u: \Sigma \rightarrow \mathbb{R}^{n}$, solves a system of the form 1

$$
-\Delta u=\Omega \cdot \nabla u \text { on } \Sigma,
$$

where $\Omega \in \operatorname{so}(n) \otimes T \Sigma$ and $\Delta$ is the negative Laplace-Beltrami operator $\frac{1}{\sqrt{|h|}} \partial_{i}\left(\sqrt{|h|} h^{i j} \partial_{j}\right)$. The fundamental fact here that has been observed in [Ri3] and exploited in this work to obtain the Hölder continuity of $W^{1,2}$-solutions to (1) is the anti-symmetry of $\Omega$.

The analysis developed in $\mathrm{Ri} 3$ permitted to generalize to general 2-dimensional conformally invariant Lagrangians the use of integrability by compensation theory as it has been introduced originally by H.Wente in the framework of constant mean curvature immersions in $\mathbb{R}^{3}$ solving the following CMC-system

$$
\Delta u=2 u_{x} \wedge u_{y} \text { on } \Sigma .
$$

Solutions to this $C M C$ system are in fact critical points to the following conformally invariant lagrangian

$$
E(u)=\frac{1}{2} \int_{\Sigma}|d u|_{h}^{2} d v o l_{h}+\int_{\Sigma} u^{*} \omega
$$

where $\omega$ is a 2 -form in $\mathbb{R}^{3}$ satisfying $d \omega=4 d x_{1} \wedge d x_{2} \wedge d x_{3}$. The natural space to consider the equation (2) is clearly the Sobolev space $W^{1,2}$. The CMC-system (2) is critical for $W^{1,2}$ in the following sense : the r.h.s. of (2) is a-priori only in $L^{1}$. Classical Calderon Zygmund theory tells us that derivatives of functions in $\Delta^{-1} L^{1}$ are in the weak $L^{2}$ space locally which is "almost" the information we started from. Hence in a sense both the quadratic non-linearity for the gradient in the r.h.s of the system and the operator in the l.h.s. are at the same level from regularity point of view and it requires a more careful analysis in order to decide which one is leading the general dynamic of this system.

H. Wente discovered the special role played by the jacobian in the r.h.s. of (2), see [He] and references therein, and was able to prove that if $u$ satisfies (2) then

$$
\|\nabla u\|_{2} \leq C\|\nabla u\|_{2}^{2}
$$

where $C$ is independent on $\Sigma$ and equals $2 \sqrt{3 / 16 \pi}$. This inequality implies that if $\sqrt{3 / 16 \pi}\|\nabla u\|_{2}<1$ then the solution is constant. This is what we call the Bootstrap Test and it is the key observation for proving Morrey estimates and deduce the Hölder regularity of general solutions to (2) which bootstraps easily in order to establish that solutions to (2) are in fact $C^{\infty}$.

Another analysis issue for this equation is to understand the behavior of sequences $u_{k}$ of solutions to the CMC system (2). Inequality (3) tells us again that if the energy does not concentrate at a point then the system will behave locally like a linear system of the form $\Delta u=0$ : the non-linearity $2 u_{x} \wedge u_{y}$ in the r.h.s is dominated by the linear highest order term $\Delta u$ in the l.h.s. . As a consequence of this fact we

\footnotetext{
${ }^{1}$ In coordinates this system reads

$$
\forall i=1 \cdots n \quad-\Delta u_{i}=\sum_{j=1}^{n} \Omega_{i}^{j} \cdot \nabla u_{j} \text { on } \Sigma
$$

where the operation is the scalar product between the gradient vector fields $\nabla u_{j}$ and the different entries of the vector valued antisymmetric matrix $\Omega$.

${ }^{2}$ This later fact has been discovered later on by Y.Ge in $\mathrm{Ge}$, see also $\mathrm{He}$.
} 
deduce that sequences of solutions to (2) with uniformly bounded energy strongly converge in $C^{p}$ norm for any $p \in \mathbb{N}$, modulo extraction of a subsequence and possibly away from finitely many point: 3 in $\Sigma$, $\left\{a_{\infty}^{1} \cdots a_{\infty}^{l}\right\}$ where the $W^{1,2}$-norm concentrates, towards a smooth limit that solves also (2)

$$
u_{k} \longrightarrow u_{\infty} \quad \text { strongly in } C_{l o c}^{p}\left(\Sigma \backslash\left\{a_{\infty}^{1} \cdots a_{\infty}^{l}\right\}\right) \quad \forall p \in \mathbb{N}
$$

The question remains to understand how the convergence at the concentration points $a_{\infty}^{i}$ fails to be strong, in other words we want to understand how and how much energy has been dissipated at the points $a_{\infty}^{i}$. A careful analysis shows that the energy is lost by concentrating solution on $\mathbb{R}^{2}$ of the CMC system (2), the so called bubbles, that converge to the $a_{\infty}^{i}$ : there exists points in $\Sigma a_{k}^{i} \rightarrow a_{\infty}^{i}$ and a familly of sequences of radii $\lambda_{k}^{i}$ converging to zero such that

$$
u_{k}\left(\lambda_{k}^{i} x+a_{k}^{i}\right) \longrightarrow \omega^{i}(x) \text { strongly in } C_{l o c}^{p}\left(\mathbb{R}^{2} \backslash\{\text { finitely many points }\}\right) \quad \forall p \in \mathbb{N} .
$$

where $\omega^{i}$ denote the bubbles, solutions on $\mathbb{R}^{2}$ of the CMC system (2). Because of the nature of the convergence it is clear that the Dirichlet energy lost in the convergences amount at least to the sum of the Dirichlet energies of the bubbles $\omega^{i}$ :

$$
\liminf _{k \rightarrow+\infty} \int_{\Sigma}\left|d u_{k}\right|_{h}^{2} d v o l_{h} \geq \int_{\Sigma}\left|d u_{\infty}\right|_{h}^{2} d v o l_{h}+\sum_{i=1}^{l} \int_{\mathbb{R}^{2}}\left|\nabla \omega^{i}\right|^{2} d x_{1} d x_{2}
$$

The question remains to understand if the inequality in (4) is strict or is in fact an equality. This question for general conformally invariant problems is known as the energy quantization question : is the loss of energy only concentrated in the forming bubbles or is there any additional dissipation in the intermediate regions between the bubbles and shrinking at the limiting concentration points $a_{\infty}^{i}$ in the so called neck region. Since the work of Sacks and Uhlenbeck [SaU] where it has been maybe first considered, in the particular framework of minimizing harmonic maps from a Riemann surface into a manifold, this question has generated a special interest, intensive researches and several detailed results have been obtained in the last decades on the subject. We refer to $[\mathrm{Ri} 2$ and reference therein for a survey on the energy quantization results. Positive results establishing energy quantization (i.e. the inequality in (4) is in fact an equality) often make use of some special geometric objects such as isoperimetric inequality or the hopf differential, see for instance [Jo] or [Pa]. In [LR1] and LR2] the second author in collaboration with F.H. Lin introduced a more functional analysis type technique based on the use of the interpolation Lorentz spaces in order to prove energy quantization results in the special cases where the non-linearity of the conformally invariant PDE can be written as a linear combination of jacobians of $W^{1,2}$-functions. Using this technique we can for instance prove that equality holds in (4) : energy quantization holds for the CMC-system, the whole loss of energy exclusively arises in the bubbles. The main step in the proof consists in using an improvement of Wente inequality (3) which has been obtained by L.Tartar and R.Coifman, P.L.Lions, Y.Meyer and S.Semmes in CLMS. This improved Lorentz-Wente type inequality reads

$$
\|\nabla u\|_{L^{2,1}} \leq C\|\nabla u\|_{2}^{2}
$$

where this time $C$ depends a-priori on $(\Sigma, h)$ and where $L^{2,1}$ denotes the Lorentz space "slightly" smaller than $L^{2}$ given by the space of measurable function $f$ on $\Sigma$ satisfying

$$
\int_{0}^{\infty} \mid\left.\{x \in \Omega \text { s.t. }|f(x)| \geq \lambda\}\right|^{\frac{1}{2}} d \lambda<+\infty \quad .
$$

The goal of the present paper is to extend energy quantization results to sequences of critical points to general conformally invariant lagrangians using functional analysis arguments in the style of [LR2].

\footnotetext{
${ }^{3}$ In our notations we can have some $a_{\infty}^{i}$ that coincide with another.
} 
The constant in the inequality (5) depends a-priori on the domain, at least on its conformal class since the equation is conformally invariant. But our neck regions connecting the bubbles are conformally equivalent to degenerating annuli. The first task of the present work is to prove different lemma which give some uniform estimates on the $L^{2,1}$-norm of the gradient for solution to Wente type equations on degenerating annuli. This is the subject of section 2 ,

In the following sections, we use these uniform estimates established in section 2 for proving various quantization phenomena. In particular we get the quantization of the angular part of the gradient for solution of general elliptic second order systems with anti-symmetric potentials. What we mean here by the angular part is the component of the gradient in the orthogonal of the radial direction with respect to the nearest point of concentration. Precisely the first main result in the present work is the following.

Theorem 0.1. Let $\Omega_{k} \in L^{2}\left(B_{1}\right.$, so $\left.(n) \otimes \mathbb{R}^{2}\right)$ and let $u_{k} \in W^{2,1}\left(B_{1}, \mathbb{R}^{n}\right)$ be a sequence of solutions of

$$
-\Delta u_{k}=\Omega_{k} \cdot \nabla u_{k}
$$

with bounded energy, i.e.

$$
\int_{B_{1}}\left(\left|\nabla u_{k}\right|^{2}+\left|\Omega_{k}\right|^{2}\right) d z \leq M
$$

Then there exists $\Omega_{\infty} \in L^{2}\left(B_{1}\right.$, so $\left.(n) \otimes \mathbb{R}^{2}\right)$ and $u_{\infty} \in W^{2,1}\left(B_{1}, \mathbb{R}^{n}\right)$ a solution of $-\Delta u_{\infty}=\Omega_{\infty} \cdot \nabla u_{\infty}$ on $B_{1}, l \in \mathbb{N}^{*}$ and

1. $\omega^{1}, \ldots, \omega^{l}$ a family of solutions to system of the form

$$
-\Delta \omega^{i}=\widetilde{\Omega}_{\infty}^{i} \cdot \nabla \omega^{i} \quad \text { on } \mathbb{R}^{2}
$$

where $\widetilde{\Omega}_{\infty}^{i} \in L^{2}\left(\mathbb{R}^{2}\right.$, so $\left.(n) \otimes \mathbb{R}^{2}\right)$,

2. $a_{k}^{1}, \ldots, a_{k}^{l}$ a family of converging sequences of points of $B_{1}$,

3. $\lambda_{k}^{1}, \ldots, \lambda_{k}^{l}$ a family of sequences of positive reals converging all to zero,

such that, up to a subsequence,

$$
\begin{gathered}
\Omega_{k} \rightarrow \Omega_{\infty} \text { in } L_{l o c}^{2}\left(B_{1}, s o(n) \otimes \mathbb{R}^{2}\right), \\
u_{k} \rightarrow u_{\infty} \text { on } W_{l o c}^{1, p}\left(B_{1} \backslash\left\{a_{\infty}^{1}, \ldots, a_{\infty}^{l}\right\}\right) \text { for all } p \geq 1
\end{gathered}
$$

and

$$
\left\|\left\langle\nabla\left(u_{k}-u_{\infty}-\sum_{i=1}^{l} \omega_{k}^{i}\right), X_{k}\right\rangle\right\|_{L_{l o c}^{2}\left(B_{1}\right)} \rightarrow 0
$$

where $\omega_{k}^{i}=\omega^{i}\left(a_{k}^{i}+\lambda_{k}^{i}.\right)$ and $X_{k}=\nabla^{\perp} d_{k}$ with $d_{k}=\min _{1 \leq i \leq l}\left(\lambda_{k}^{i}+d\left(a_{k}^{i},.\right)\right)$.

Moreover, if we have $\left\|\Omega_{k}\right\|_{\infty}=O(1)$ or even just $\Omega_{k}=O\left(\left|\nabla u_{k}\right|\right)$ hence the convergence to the limit solution $u_{\infty}$ is in fact in $C_{l o c}^{1, \eta}$ for all $\eta \in[0,1[$.

The proof of theorem 0.1 is established through the iteration of the following result. It says that, if the $L^{2}$ norm of the potential $\Omega$ is below some threshold on every dyadic sub-annuli of a given annulus, the angular part of the Dirichlet energy of $u$ on a slightly smaller annulus is controlled by the maximal contribution of the Dirichlet energy of $u$ on the dyadic sub-annuli. Precisely we prove the following. 
Theorem 0.2. There exists $\delta>0$ such that for all $r, R \in \mathbb{R}_{+}^{*}$ satisfying $2 r<R$ for all $\Omega \in L^{2}\left(B_{R} \backslash\right.$ $\left.B_{r}, s o(n) \otimes \mathbb{R}^{n}\right)$ and $u \in W^{1,2}\left(B_{R} \backslash B_{r}, \mathbb{R}^{n}\right)$ satisfying

$$
-\Delta u=\Omega \cdot \nabla u
$$

and

$$
\sup _{r<\rho<\frac{R}{2}} \int_{B_{2 \rho} \backslash B_{\rho}}|\Omega|^{2} d z \leq \delta .
$$

Then there exists $C>0$, independent of $u, r$ and $R$, such that

$$
\left\|\frac{1}{\rho} \frac{\partial u}{\partial \theta}\right\|_{L^{2}\left(B_{\frac{R}{2} \backslash B_{2 r}}\right)}^{2} \leq C\|\nabla u\|_{2}\left[\sup _{r<\rho<\frac{R}{2}} \int_{B_{2 \rho} \backslash B_{\rho}}|\nabla u|^{2} d z\right]^{1 / 2} .
$$

Thanks to the quantization of the angular part for general elliptic systems with anti-symmetric potential, we can derive the energy quantization for critical points to an arbitrary continuously conformally invariant elliptic Lagrangian with quadratic growth.

Theorem 0.3. Let $N^{k}$ be a $C^{2}$ submanifold of $\mathbb{R}^{m}$ and $\omega$ be a $C^{1} 2$-form on $N^{k}$ such that the $L^{\infty}$-norm of $d \omega$ is bounded on $N^{k}$. Let $u_{k}$ be a sequence of critical points in $W^{1,2}\left(B_{1}, N^{k}\right)$ for the Lagrangian

$$
F(u)=\int_{B_{1}}\left[|\nabla u|^{2}+\omega(u)\left(u_{x}, u_{y}\right)\right] d z
$$

with uniformly bounded energy, i.e.

$$
\left\|\nabla u_{k}\right\|_{2} \leq M
$$

Then there exists $\Lambda \in C^{0}\left(T N \otimes \mathbb{R}^{2}\right.$, so $\left.(n) \otimes \mathbb{R}^{2}\right)$ and $u_{\infty} \in W^{1,2}\left(B_{1}, \mathbb{R}^{n}\right)$ a solution of $-\Delta u=\Lambda(u, \nabla u) \cdot \nabla u$ on $B_{1}, l \in \mathbb{N}^{*}$ and

1. $\omega^{1}, \ldots, \omega^{l}$ some non-constant $\Lambda$-bubbles, i.e non-constant solution of

$$
-\Delta \omega=\Lambda(\omega, \nabla \omega) \cdot \nabla \omega \text { on } \mathbb{R}^{2}
$$

2. $a_{k}^{1}, \ldots, a_{k}^{l}$ a family of converging sequences of points of $B_{1}$,

3. $\lambda_{k}^{1}, \ldots, \lambda_{k}^{l}$ a family of sequences of positive reals converging all to zero,

such that, up to a subsequence,

$$
u_{k} \rightarrow u_{\infty} \text { on } C_{l o c}^{1, \eta}\left(B_{1} \backslash\left\{a_{\infty}^{1}, \ldots, a_{\infty}^{l}\right\}\right) \text { for all } \eta \in[0,1[
$$

and

where $\omega_{k}^{i}=\omega^{i}\left(a_{k}^{i}+\lambda_{k}^{i} \cdot\right)$.

$$
\left\|\nabla\left(u_{k}-u_{\infty}-\sum_{i=1}^{l} \omega_{k}^{i}\right)\right\|_{L_{l o c}^{2}\left(B_{1}\right)} \rightarrow 0
$$

Previous works establishing energy quantizations for various conformally invariant elliptic Lagrangian usually require more regularity on the Lagrangian ( see for instance [Jo, [Pa, [St, $\mathrm{DiT}], \mathrm{LiWa}, \mathrm{Zhu}$ ). For instance in $\mathrm{Pa}$ or $[\mathrm{LiWa}$ the energy quantization for harmonic maps in two dimension is obtained through the application of the maximum principle to an ordinary differential inequality satisfied by the integration over concentric circles of the angular part of the energy. The application of this procedure required an $L^{\infty}$ bound on the derivatives of the second fundamental form, see lemma 2.1 of [LiWa]. We 
insist on the fact that, in comparison to the previously existing energy quantization results, theorem 0.3 above requires an $C^{0}$ bound on the second fundamental form only, which is a weakening of the regularity assumption for the target of a magnitude one with respect to derivation. Another application of theorem 0.3 is the energy quantization for solutions to the prescribed mean curvature system, see corollary 4.1. assuming only an $C^{0}$ bound on the mean curvature. Again, previous energy quantization results were assuming uniform $C^{1}$ bounds on $H$, see [BeRe] and $\mathrm{CaMu}$. Theorem 0.3 in the prescribed mean curvature system corresponds again for this problem to weakening of the regularity assumption for the target of a magnitude one with respect to derivation in comparison to previous existing result . This weaker assumption moreover are the minimal one in order for the Lagrangian to be continuously differentiable and this is why it coincides with the original one appearing in the formulation of the Heinz-Hildebrandt regularity conjecture in the 70 's.

In a last part, we present some more applications of the uniform Lorentz-Wente estimates we established in section 2. The first one, for instance, deals with sequences of pseudo holomorphic immersions of sequences of closed Riemann surfaces whose corresponding conformal class degenerate in the moduli space of the underlying 2-dimensional manifold. In particular we give a new proof of the Gromov's compactness theorem in all generality, see theorem 5.1. We also give some cohomological condition which garanties the energy quantization for sequences of harmonic maps on degenerating surfaces. Finally we give a very brief introduction to the quantization of the Willmore surface established recently in $[\overline{B R}$, where the uniform Lorentz-Wente estimates of section 2 play a crucial role.

Acknowledgements: The first author was visiting the Forschungsinstituts für Mathematik at E.T.H. (Zurich) when this work started, he would like to thank it for its hospitality and the excellent working conditions. The two authors would like to thank Francesca Da Lio for her useful comments on the manuscript.

\section{Lorentz spaces and standard Wente's inequalities}

Lorentz spaces seems to be the good spaces in order to get precise Wente's inequality, here we recall some classical facts about these spaces, StWe and Gra] for details.

Definition 1.1. Let $D$ be a domain of $\left.\mathbb{R}^{k}, p \in\right] 1,+\infty\left[\right.$ and $q \in[1,+\infty]$. The Lorentz space $L^{p, q}(D)$ is the set of measurable functions $f: D \rightarrow \mathbb{R}$ such that

$$
\|f\|_{p, q}=\left(\int_{0}^{+\infty}\left(t^{\frac{1}{p}} f^{* *}(t)\right)^{q} \frac{d t}{t}\right)^{\frac{1}{q}}<+\infty \text { if } q<+\infty
$$

or

$$
\|f\|_{p, \infty}=\sup \left(t^{\frac{1}{p}} f^{* *}(t)\right) \text { if } q=+\infty
$$

where $f^{* *}(t)=\frac{1}{t} \int_{0}^{t} f^{*}(s) d s$ and $f^{*}$ the decreasing rearrangement of $f$.

Each $L^{p, q}$ may be seen as a deformation of $L^{p}$. For instance, we have the strict inclusions

$$
L^{p, 1} \subset L^{p, q^{\prime}} \subset L^{p, q^{\prime \prime}} \subset L^{p, \infty},
$$

if $1<q^{\prime}<q^{\prime \prime}$. Moreover,

$$
L^{p, p}=L^{p}
$$

Furthermore, if $|D|$ is finite, we have that for all $q$ and $q^{\prime}$,

$$
p>p^{\prime} \Rightarrow L^{p, q} \subset L^{p^{\prime}, q^{\prime}} .
$$


Finally, for $p \in] 1,+\infty\left[\right.$ and $q \in[1,+\infty], L^{\frac{p}{p-1}, \frac{q}{q-1}}$ is the dual of $L^{p, q}$.

In the case $p, q=2,1$ we can give an other definition. Let $\phi(\lambda)=\mid\left\{t \in[0,|D|]\right.$ s.t. $\left.f^{*}(t) \geq \lambda\right\} \mid$, we make the change of variable $t=\phi(\lambda)$ in the definition of the Lorentz-norm, which gives

$$
\|f\|_{2,1}=2 \int_{\sup |f|}^{0} \phi^{-\frac{1}{2}}(\lambda) \lambda \phi^{\prime}(\lambda) d \lambda .
$$

Hence integrating by part, we get

$$
\|f\|_{2,1}=4 \int_{0}^{+\infty} \mid\left.\{x \in \Omega \text { s.t. }|f(x)| \geq \lambda\}\right|^{\frac{1}{2}} d \lambda .
$$

To finish this preliminary, we quickly present the standard Wente's inequalities for elliptic system in Jacobian form. Indeed if $a$ and $b$ are in $W^{1,2}$ this is clear that $a_{x} b_{y}-a_{y} b_{x}$ is in $L^{1}$ but in fact thanks to its structure, it is subject to compensated phenomena and $a_{x} b_{y}-a_{y} b_{x}$ is in $\mathcal{H}^{1}$ the Hardy space which is a strict subspace of $L^{1}$ which as a better behaviour than $L^{1}$ with respect to Calderon-Zygmund theory, since the convolution of a function in $\mathcal{H}^{1}$ and the Green kernel $\log (|z|)$ is in $W^{2,1}$. This improvement of integrability is summarized in the following theorem.

Lemma 1.1 (We, , Tar, , [LMS] $)$. Let $a$ and $b$ be in $W^{1,2}\left(B_{1}\right)$. Let $\phi \in W_{0}^{1,1}\left(B_{1}\right)$ be the solution of

$$
\Delta \phi=a_{x} b_{y}-a_{y} b_{x} \text { on } B_{1}
$$

Then there exists a constant $C$ independent of $\phi$ such that

$$
\|\phi\|_{\infty}+\|\nabla \phi\|_{2,1}+\left\|\nabla^{2} \phi\right\|_{1} \leq C\|\nabla a\|\|\nabla b\|_{2} .
$$

A consequence of the previous theorem was obtain by Bethuel Bet using a duality argument.

Lemma 1.2. Let $a$ and $b$ be two measurable functions such that $\nabla a \in L^{2, \infty}\left(B_{1}\right)$ and $\nabla b \in L^{2}\left(B_{1}\right)$. Let $\phi \in W_{0}^{1,1}\left(B_{1}\right)$ be the solution of

$$
\Delta \phi=a_{x} b_{y}-a_{y} b_{x} \text { on } B_{1}
$$

Then there exists a constant $C$ independent of $\phi$ such that

$$
\|\nabla \phi\|_{2} \leq C\|\nabla a\|_{2, \infty}\|\nabla b\|_{2} .
$$

Notation: In the following, if we consider a norm with out specified its domains, it is implicitly assume that its domain of definition is the one of the function. We denote $B_{R}(p)$ the ball of radius $R$ centered at $p$ and we just denote $B_{R}$ when $p=0$.

\section{$2 \quad$ Wente type lemmas}

In this section we are going to prove some uniform Wente's estimates on annuli whose conformal class is a priori not bounded. In fact those estimate were already known for the $L^{\infty}$-norm and the $L^{2}$-norm of the gradient, since it has been proved that the constant is in fact independent of the domain considered, see $\left[\mathrm{To}\right.$ and $\left[\mathrm{Ge}\right.$. But this fact is to our knowledge new for the $L^{2,1}$-norm of the gradient.

Lemma 2.1. let $a, b \in W^{1,2}\left(B_{1}\right), 0<\varepsilon<\frac{1}{2}$, and $\phi \in W_{0}^{1,1}\left(B_{1} \backslash B_{\varepsilon}\right)$ a solution of

$$
\Delta \phi=a_{x} b_{y}-a_{y} b_{x} \text { on } B_{1} \backslash B_{\varepsilon} .
$$

Then $\nabla \phi \in L^{2,1}\left(B_{1} \backslash B_{\varepsilon}\right)$ and, for all $\lambda>1$, there exists a positive constant $C(\lambda)$ independent of $\varepsilon$ and $\phi$ such that

$$
\|\nabla \phi\|_{L^{2,1}\left(B_{1} \backslash B_{\lambda \varepsilon}\right)} \leq C(\lambda)\|\nabla a\|_{2}\|\nabla b\|_{2} .
$$




\section{Proof of lemma 2.1:}

First we consider a solution of our equation on the whole disk, that is to say $\varphi \in W_{0}^{1,1}\left(B_{1}\right)$ which satisfies

$$
\Delta \varphi=a_{x} b_{y}-a_{y} b_{x} \text { on } B_{1} .
$$

Then thanks to the classical Wente's inequality (10), we have

$$
\|\varphi\|_{\infty}+\|\nabla \varphi\|_{2,1} \leq C\|\nabla a\|_{2}\|\nabla b\|_{2} \cdot
$$

where $C$ is a positive constant independent of $\varphi$.

Then we set $\psi=\phi-\varphi$, which satisfies

$$
\left\{\begin{array}{l}
\Delta \psi=0 \text { on } B_{1} \backslash B_{\varepsilon} \\
\psi=0 \text { on } \partial B_{1} \\
\psi=-\varphi \text { on } \partial B_{\varepsilon}
\end{array}\right.
$$

Hence $\widetilde{\psi}=\psi-\left(\int_{\partial B_{\varepsilon}} \psi d \sigma\right) \frac{\ln (|z|)}{2 \pi \varepsilon \ln (\varepsilon)}$ satisfies the hypothesis of the lemma A.1, then

$$
\|\nabla \widetilde{\psi}\|_{L^{2,1}\left(B_{1} \backslash B_{\lambda \varepsilon}\right)} \leq C(\lambda)\|\nabla \widetilde{\psi}\|_{2} \text { for all } \lambda>1 \text {. }
$$

Hence, computing the $L^{2}$-norm of the gradient of the logarithm on $B_{1} \backslash B_{\lambda \varepsilon}$, we get that

$$
\|\nabla \widetilde{\psi}\|_{L^{2,1}\left(B_{1} \backslash B_{\lambda \varepsilon}\right)} \leq C(\lambda)\left(\|\nabla \psi\|_{2}+\left(\int_{\partial B_{\varepsilon}}|\psi| d \sigma\right) \frac{1}{\varepsilon \sqrt{\ln \left(\frac{1}{\varepsilon}\right)}}\right) .
$$

But $\psi$ is the harmonic on $B_{1} \backslash B_{\varepsilon}$ and is equal to $-\varphi$ on the boundary, then

$$
\|\nabla \psi\|_{2} \leq\|\nabla \varphi\|_{2} \text { and }\|\psi\|_{\infty} \leq\|\varphi\|_{\infty} .
$$

Hence we get that

$$
\int_{\partial B_{\varepsilon}}|\psi| d \sigma \leq \varepsilon C(\lambda)\|\nabla a\|_{2}\|\nabla b\|_{2},
$$

which gives, using (13) and (14), that

$$
\|\nabla \widetilde{\psi}\|_{L^{2,1}\left(B_{1} \backslash B_{\lambda \varepsilon}\right)} \leq C(\lambda)\|\nabla a\|_{2}\|\nabla b\|_{2} .
$$

Finally, computing the $L^{2,1}$-norm of the gradient of the logarithm on $B_{1} \backslash B_{\lambda \varepsilon}$, we get that

$$
\|\nabla \ln \|_{L^{2,1}\left(B_{1} \backslash B_{\lambda \varepsilon}\right)}=4 \sqrt{\pi} \ln \left(\frac{1}{\lambda \varepsilon}\right) .
$$

Hence, thanks to (15), (16) and (17), we get that

$$
\|\nabla \psi\|_{L^{2,1}\left(B_{1} \backslash B_{\lambda \varepsilon}\right)} \leq C(\lambda)\|\nabla a\|_{2}\|\nabla b\|_{2} .
$$

Then, thanks to (12) and (18), we get the desired estimate. 
Lemma 2.2. let $a, b \in W^{1,2}\left(B_{1}\right), 0<\varepsilon<\frac{1}{4}$, and $\phi \in W^{1,1}\left(B_{1} \backslash B_{\varepsilon}\right)$ a solution of

$$
\left\{\begin{array}{l}
\Delta \phi=a_{x} b_{y}-a_{y} b_{x} \text { on } B_{1} \backslash B_{\varepsilon} \\
\int_{\partial B_{\varepsilon}} \phi d \sigma=0, \\
\left|\int_{\partial B_{1}} \phi d \sigma\right| \leq K,
\end{array}\right.
$$

where $K$ is a constant independent of $\varepsilon$. Then, for all $0<\lambda<1$, there exists a positive constant $C(\lambda)$ independent of $\varepsilon$ such that

$$
\|\nabla \phi\|_{L^{2,1}\left(B_{\lambda} \backslash B_{\lambda-1}\right)} \leq C(\lambda)\left(\|\nabla a\|_{2}\|\nabla b\|_{2}+\|\nabla \phi\|_{2}+1\right)
$$

Proof of lemma 2.2. :

Let $u \in W^{1,1}\left(B_{1} \backslash B_{\varepsilon}\right)$ be the solution of

$$
\left\{\begin{array}{l}
\Delta u=0 \text { on } B_{1} \backslash B_{\varepsilon}, \\
u=\phi \text { on } \partial B_{1} \cup \partial B_{\varepsilon} .
\end{array}\right.
$$

Hence $\|\nabla u\|_{2} \leq\|\nabla \phi\|_{2}$. Moreover thanks to lemma A.2 and lemma 2.1 we have $\nabla u \in L^{2,1}\left(B_{\lambda} \backslash B_{\lambda^{-1} \varepsilon}\right)$ and $\nabla(u-\phi) \in L^{2,1}\left(B_{1} \backslash B_{\lambda^{-1} \varepsilon}\right)$ with

$$
\begin{aligned}
& \|\nabla u\|_{L^{2,1}\left(B_{\lambda} \backslash B_{\lambda}-1_{\varepsilon}\right.} \leq C(\lambda)\left(\|\nabla \phi\|_{2}+1\right) \\
& \|\nabla(u-\phi)\|_{L^{2,1}\left(B_{\lambda} \backslash B_{\lambda}-1_{\varepsilon}\right)} \leq C(\lambda)\|\nabla a\|_{2}\|\nabla b\|_{2},
\end{aligned}
$$

which proves lemma 2.2

Remark: As in lemma A.2 we cannot control the $L^{2,1}$-norm of $\nabla \phi$ by its $L^{2}$-norm, as it is shown by the following example

$$
z \mapsto \frac{\ln \left(\frac{|z|}{\varepsilon}\right)}{\ln \left(\frac{1}{\varepsilon}\right)} .
$$

Lemma 2.3. let $a, b \in W^{1,2}\left(B_{1}\right), 0<\varepsilon<\frac{1}{4}$ and $\phi \in W^{1,2}\left(B_{1} \backslash B_{\varepsilon}\right)$ be a solution of

$$
\Delta \phi=a_{x} b_{y}-a_{y} b_{x} \text { on } B_{1} \backslash B_{\varepsilon} .
$$

Moreover, we assume that

$$
\|\phi\|_{\infty}<+\infty
$$

Then, for $0<\lambda<1$ a positive constant $C(\lambda)$ independent of $\varepsilon$ and $\phi$ such that

$$
\|\nabla \phi\|_{L^{2,1}\left(B_{\lambda} \backslash B_{\lambda-1}\right)} \leq C(\lambda)\left(\|\nabla a\|_{2}\|\nabla b\|_{2}+\|\phi\|_{\infty}\right) .
$$




\section{Proof of lemma 2.3. :}

We introduce first $\varphi \in W_{0}^{1,2}\left(B_{1} \backslash B_{\varepsilon}\right)$ to be the unique solution to

$$
\left\{\begin{array}{l}
\Delta \varphi=a_{x} b_{y}-a_{y} b_{x} \text { on } B_{1} \backslash B_{\varepsilon} \\
\varphi=0 \text { on } \partial B_{1} \cup \partial B_{\varepsilon} .
\end{array}\right.
$$

Then thanks to lemma 2.1, we have

$$
\|\nabla \varphi\|_{L^{2,1}\left(B_{1} \backslash B_{\lambda-1}\right)} \leq C(\lambda)\|\nabla a\|_{2}\|\nabla b\|_{2},
$$

where $C(\lambda)$ is a positive constant depending on $\lambda$ but not on $\phi$ and $\varepsilon$.

Then we set $\psi=\phi-\varphi$, which is harmonic. Thanks to standard estimates on harmonic function, see HaLi] for instance, there exists $C(\lambda)>0$ a positive constant independent of $\psi$ and $\varepsilon$ such that

$$
\|\psi\|_{L^{2,1}\left(B_{\lambda} \backslash B_{\lambda-1}\right)} \leq C(\lambda)\|\psi\|_{L^{\infty}\left(\partial B_{1} \cup \partial B_{\varepsilon}\right)} \leq C\|\phi\|_{L^{\infty}} .
$$

Which proves the desired inequality and lemma 2.3 is proved.

Lemma 2.4. let $a, b \in L^{2}\left(B_{1}\right), 0<\varepsilon<\frac{1}{4}$, assume that $\nabla a \in L^{2, \infty}\left(B_{1}\right)$ and that $\nabla b \in L^{2}\left(B_{1}\right)$, let $\phi \in W^{1,(2, \infty)}\left(B_{1} \backslash B_{\varepsilon}\right)$ a solution of

$$
\Delta \phi=a_{x} b_{y}-a_{y} b_{x} \text { on } B_{1} \backslash B_{\varepsilon},
$$

Denote, for $\varepsilon \leq r \leq 1, \phi_{0}(r):=(2 \pi r)^{-1} \int_{\partial B_{r}(0)} \phi d \sigma$ and assume

$$
\int_{\varepsilon}^{1}\left|\dot{\phi}_{0}\right|^{2} r d r<+\infty
$$

Then, for $0 \leq \lambda<1$, there exists a positive constant $C(\lambda)>0$ independent of $\varepsilon$ and $\phi$ such that

$$
\begin{aligned}
\|\nabla \phi\|_{L^{2}\left(B_{\lambda} \backslash B_{\lambda-1}\right)} & \leq C(\lambda)\left(\|\nabla a\|_{2, \infty}\|\nabla b\|_{2}+\left\|\nabla \phi_{0}\right\|_{L^{2}\left(B_{1} \backslash B_{\varepsilon}\right)}\right. \\
& \left.+\|\nabla \phi\|_{L^{2, \infty}\left(B_{1} \backslash B_{\varepsilon}\right)}\right) .
\end{aligned}
$$

Proof of lemma 2.4:

First we consider $\varphi \in W_{0}^{1,2}\left(B_{1}\right)$ to be the solution of

$$
\left\{\begin{array}{l}
\Delta \varphi=a_{x} b_{y}-a_{y} b_{x} \text { on } B_{1} \\
\varphi=0 \quad \text { on } \partial B_{1}
\end{array} .\right.
$$

Then thanks to the generalized Wente's inequality, see (11), we have

$$
\|\nabla \varphi\|_{2} \leq C\|\nabla a\|_{2, \infty}\|\nabla b\|_{2} \quad .
$$

Consider the difference $v:=\phi-\varphi-\left(\phi_{0}-\varphi_{0}\right)$, it is an harmonic function on $B_{1} \backslash B_{\varepsilon}$ which does not have 0 -frequency Fourier modes :

$$
v=\sum_{n \in \mathbb{Z}^{*}}\left(c_{n} \rho^{n}+d_{n} \rho^{-n}\right) e^{i n \theta}
$$


which implies in particular that

$$
\int_{\partial B_{\rho}} \frac{\partial v}{\partial \nu} d \sigma=0 \text { for all } \varepsilon<\rho<1 .
$$

Moreover, due to the assumption (24) and due to (26) we have

$$
\begin{aligned}
\|\nabla v\|_{L^{2, \infty}\left(B_{1} \backslash B_{\varepsilon}\right)} & \leq 2\|\nabla \varphi\|_{2}+\left\|\nabla \phi_{0}\right\|_{2}+\|\nabla \phi\|_{L^{2, \infty}\left(B_{1} \backslash B_{\varepsilon}\right)} \\
& \leq C\left(\|\nabla a\|_{2, \infty}\|\nabla b\|_{2}+\left\|\nabla \phi_{0}\right\|_{2}+\|\nabla \phi\|_{L^{2, \infty}\left(B_{1} \backslash B_{\varepsilon}\right)}\right)
\end{aligned}
$$

Let $\lambda \in] 0,1\left[\right.$, then standard elliptic estimates on harmonic function give that $\forall \rho \in\left(\lambda^{-1} \varepsilon, \lambda\right)$

$$
\begin{aligned}
& \|\nabla v\|_{L^{\infty}\left(\partial B_{\rho}\right)} \leq C(\lambda) \rho^{-1}\|\nabla v\|_{L^{2, \infty}\left(B_{\lambda-1} \backslash B_{\lambda \rho}\right)} \\
& \quad \leq C(\lambda) \rho^{-1}\left(\|\nabla a\|_{2, \infty}\|\nabla b\|_{2}+\left\|\nabla \phi_{0}\right\|_{2}+\|\nabla \phi\|_{L^{2, \infty}\left(B_{1} \backslash B_{\varepsilon}\right)}\right)
\end{aligned}
$$

Denote $\Omega_{\varepsilon}:=B_{\lambda} \backslash B_{\lambda^{-1} \varepsilon}$. We have that

$$
\|\nabla v\|_{L^{2}\left(\Omega_{\varepsilon}\right)}=\sup _{\left\{X ;\|X\|_{L^{2}\left(\Omega_{\varepsilon}\right)} \leq 1\right\}} \int_{\Omega_{\varepsilon}} \nabla v \cdot X d z
$$

For such an $X \in L^{2}\left(\Omega_{\varepsilon}\right)$ we denote $\widetilde{X}$ it's extension by 0 in the complement of $\Omega_{\varepsilon}$ in $B_{1}$. Let $g$ be the solution of

$$
\left\{\begin{array}{l}
\Delta g=-\operatorname{div} \tilde{X}^{\perp} \quad \text { in } B_{1} \\
g=0 \quad \text { on } \partial B_{1}
\end{array}\right.
$$

where $\widetilde{X}^{\perp}=\left(-\widetilde{X}_{2}, \widetilde{X}_{1}\right)$. We easily see that

$$
\|\nabla g\|_{L^{2}\left(B_{1}\right)} \leq C\|\tilde{X}\|_{L^{2}\left(B_{1}\right)} \leq C .
$$

Poincaré lemma gives the existence of $f \in W^{1,2}\left(B_{1}\right)$ such that

$$
\widetilde{X}=\nabla f+\nabla^{\perp} g
$$

and we have

$$
\|\nabla f\|_{L^{2}\left(B_{1}\right)} \leq\|\nabla g\|_{L^{2}\left(B_{1}\right)}+\|\widetilde{X}\|_{L^{2}\left(B_{1}\right)}+\leq C+1
$$

We have

$$
\int_{\Omega_{\varepsilon}} \nabla v \cdot X d z=\int_{\Omega_{\varepsilon}} \nabla v \cdot \nabla f d z+\int_{\Omega_{\varepsilon}} \nabla v \cdot \nabla^{\perp} g d z
$$

We write

$$
\begin{aligned}
\int_{\Omega_{\varepsilon}} & \nabla v \cdot \nabla^{\perp} g d z=\int_{\partial B_{\lambda}} \partial_{\tau} v g d \sigma-\int_{\partial B_{\lambda^{-1} \varepsilon}} \partial_{\tau} v g d \sigma \\
= & \int_{\partial B_{\lambda}} \partial_{\tau} v\left(g-g_{\lambda}\right) d \sigma-\int_{\partial B_{\lambda^{-1} 1_{\varepsilon}}} \partial_{\tau} v\left(g-g_{\lambda^{-1} \varepsilon}\right) d \sigma
\end{aligned}
$$

where $\partial_{\tau}$ is the tangential derivative along the circles $\partial B_{\lambda}$ and $\partial B_{\lambda^{-1} \varepsilon}$ and $g_{\lambda}$ (resp. $g_{\lambda^{-1}}$ ) denote the average of $g$ on $\partial B_{\lambda}$ (resp. $\left.B_{\lambda^{-1} \varepsilon}\right)$. 
We have for any $\rho \in(0,1)$

$$
\frac{1}{\rho} \int_{\partial B_{\rho}}\left|g-g_{\rho}\right| d \sigma \leq C\|g\|_{H^{1 / 2}\left(\partial B_{\rho}\right)} \leq C\|\nabla g\|_{2} \leq C
$$

where $C$ is independent of $\rho$. Combining (29), (34) and (33) give in one hand

$$
\left|\int_{\Omega_{\varepsilon}} \nabla v \cdot \nabla^{\perp} g\right| d z \leq C(\lambda)\|\nabla v\|_{L^{2, \infty}\left(B_{1} \backslash B_{\varepsilon}\right)} .
$$

In the other hand one using the fact that $v$ is harmonic and satisfies (27) we have

$$
\begin{aligned}
\int_{\Omega_{\varepsilon}} & \nabla v \cdot \nabla f=\int_{\partial B_{\lambda}} \partial_{\nu} v f-\int_{\partial B_{\lambda}-1_{\varepsilon}} \partial_{\nu} v f \\
= & \int_{\partial B_{\lambda}} \partial_{\nu} v\left(f-f_{\lambda}\right)-\int_{\partial B_{\lambda-1} \varepsilon} \partial_{\nu} v\left(f-f_{\lambda^{-1} \varepsilon}\right)
\end{aligned}
$$

We have for any $\rho \in(0,1)$

$$
\frac{1}{\rho} \int_{\partial B_{\rho}}\left|f-f_{\rho}\right| \leq C\|f\|_{H^{1 / 2}\left(\partial B_{\rho}\right)} \leq C\|\nabla f\|_{2} \leq C
$$

Combining now (29), (36) together with (37) we obtain

$$
\left|\int_{\Omega_{\varepsilon}} \nabla v \cdot \nabla f\right| \leq C(\lambda)\|\nabla v\|_{L^{2, \infty}\left(B_{1} \backslash B_{\varepsilon}\right)} .
$$

Combining (35), (38) with (30) gives

$$
\|\nabla v\|_{L^{2}\left(\Omega_{\varepsilon}\right)} \leq C(\lambda)\|\nabla v\|_{L^{2, \infty}\left(B_{1} \backslash B_{\varepsilon}\right)}
$$

This inequality together with (24), (26) gives (25) and the lemma is proved.

\section{Angular Energy Quantization for solutions to elliptic systems with anti-symmetric potential}

The aim of this section is to prove that the angular part of the gradient of a bounded sequence of solutions of an elliptic system with anti-symmetric potential is always quantified. But before starting the proof of the quantization, we remind some fact about elliptic systems with antisymmetric potential which have intensively studied by the second author [Ri3].

Let $\Omega \in L^{2}\left(B_{1}, s o(n) \otimes \mathbb{R}^{n}\right)$ we consider $u \in W^{1,2}\left(B_{1}, \mathbb{R}^{n}\right)$ a solution of the following equation

$$
-\Delta u=\Omega \cdot \nabla u \text { on } B_{1} \text {. }
$$

One of the fundamental fact about this system is the discover a conservation law using a Coulomb gauge for $\Omega$ when its $L^{2}$-norm is small enough which is the aim of the following theorem.

Theorem 3.1 (Theorem I.4 [Ri3]). There exists $\varepsilon_{0}>0$ such that for all $\Omega \in L^{2}\left(B_{1}\right.$, so $\left.(n) \otimes \mathbb{R}^{2}\right)$ satisfying

$$
\int_{B_{1}}|\Omega|^{2} d z \leq \varepsilon_{0}
$$


then there exists $A \in W^{1,2} \cap L^{\infty}\left(B_{1}, G l_{n}(\mathbb{R})\right)$ such that

$$
\operatorname{div}(\nabla A-A \Omega)=0
$$

and

$$
\int_{B_{1}}\left(|\nabla A|^{2}+\left|\nabla A^{-1}\right|^{2}\right) d z+\operatorname{dist}\left(\left\{A, A^{-1}\right\}, S O(n)\right) \leq C \int_{B_{1}}|\Omega|^{2} d z,
$$

where $C$ is a constant independent of $\Omega$. that

Then, using this theorem and Poincaré's lemma, we get the existence of $B \in W^{1,2}\left(B_{1}, M_{n}(\mathbb{R})\right)$ such

$$
\operatorname{div}(A \nabla u)=\nabla^{\perp} B \cdot \nabla u
$$

and

$$
\int_{B_{1}}|\nabla B|^{2} d z \leq C \int_{B_{1}}|\Omega|^{2} d z .
$$

Hence the system is rewrite in Jacobian form and we can use standard Wente's estimates. In particular, this permits to prove three fundamental properties of the solutions of this equation which are the $\varepsilon$ regularity, the energy gap for solution defined on the whole plane and the passage to the weak limit in the equation. This properties are summarized in the following theorem.

Theorem 3.2. [Ri3], [Ri6] There exists $\varepsilon_{0}>0$ and $C_{q}>0$, depending only on $q \in \mathbb{N}^{*}$, such that if $\Omega \in L^{2}\left(B_{1}\right.$, so $\left.(n) \otimes \mathbb{R}^{2}\right)$ (reps. $L^{2}\left(\mathbb{R}^{2}\right.$, so $\left.(n) \otimes \mathbb{R}^{2}\right)$ ) satisfies

$$
\|\Omega\|_{2}^{2} \leq \varepsilon_{0}
$$

then

1. (E-regularity) If $u \in W^{1,2}\left(B_{1}, \mathbb{R}^{n}\right)$ satisfies

$$
-\Delta u=\Omega \cdot \nabla u \text { on } B_{1}
$$

then we have the following estimate

$$
\|\nabla u\|_{L^{q}\left(B_{\frac{1}{4}}\right)} \leq C_{q}\|\nabla u\|_{2} \text { for all } q \in \mathbb{N}^{*}
$$

2. (Energy gap) If $u \in W^{1,2}\left(\mathbb{R}^{2}, \mathbb{R}^{n}\right)$ satisfies

$$
-\Delta u=\Omega \cdot \nabla u \text { on } \mathbb{R}^{2}
$$

then it is constant.

3. (Weak limit property) Let $\Omega_{k} \in L^{2}\left(B_{1}, s o(n) \otimes \mathbb{R}^{2}\right)$ such that $\Omega_{k}$ weakly converge in $L^{2}$ to $\Omega$ and $u_{k}$ a bounded sequence in $W^{1,2}\left(B_{1}, \mathbb{R}^{n}\right)$ which satisfies

$$
-\Delta u_{k}=\Omega_{k} \cdot \nabla u_{k} \text { on } B_{1}
$$

Then, there exists a subsequence of $u_{k}$ which weakly converge in $W^{1,2}\left(B_{1}, \mathbb{R}^{n}\right)$ to a solution of

$$
-\Delta u=\Omega \cdot \nabla u \text { on } B_{1} .
$$


For the convenience of the reader we recall the arguments developed in [ $\mathrm{Ri} 3$ ] and [Ri6] to prove theorem 3.2

\section{Proof of theorem 3.2 :}

In order to prove the $\varepsilon$-regularity, let us prove that, for $\alpha>0$, we have

$$
\sup _{p \in B_{1 / 2}, 0<\rho<\frac{1}{2}} \rho^{-\alpha} \int_{B_{\rho}(p)}|\Delta u| d z \leq C\|\nabla u\|_{L^{2}\left(B_{1}\right)} .
$$

A classical estimate on Riesz potentials gives

$$
|\nabla u|(p) \leq C \frac{1}{|x|} * \chi_{B_{1 / 2}}|\Delta u|+C\|\nabla u\|_{L^{2}\left(B_{1}\right)} \quad \forall p \in B_{1 / 4},
$$

where $\chi_{B_{1 / 2}}$ is the characteristic function of the ball $B_{\frac{1}{2}}$. Together with injections proved by Adams in Ad], the latter shows that

$$
\|\nabla u\|_{L^{r}\left(B_{\frac{1}{4}}\right)} \leq C\|\nabla u\|_{L^{2}\left(B_{1}\right)}
$$

for some $r>1$. Then bootstrapping this estimate, see lemma IV.1 of [Ri6] or theorem 1.1 of [ShTo], we get

$$
\|\nabla u\|_{L^{q}\left(B_{\frac{1}{4}}\right)} \leq C_{q}\|\nabla u\|_{L^{2}\left(B_{1}\right)} \text { for all } q \in \mathbb{N}^{*},
$$

which will prove the $\varepsilon$-regularity.

In order to prove (43), we assume that $\varepsilon_{0}$ is small enough to apply theorem 3.1]. Hence there exists $A \in W^{1,2} \cap L^{\infty}\left(B_{1}, G l_{n}(\mathbb{R})\right)$ and $B \in W^{1,2} \cap L^{\infty}\left(B_{1}, M_{n}(\mathbb{R})\right)$ such that

$$
\int_{B_{1}}\left(|\nabla A|^{2}+|\nabla B|^{2}\right) d z+\operatorname{dist}\left(\left\{A, A^{-1}\right\}, S O(n)\right) \leq C \int_{B_{1}}|\Omega|^{2} d z .
$$

and

$$
\left\{\begin{array}{l}
\operatorname{div}(A \nabla u)=\nabla^{\perp} B \cdot \nabla u, \\
\operatorname{curl}(A \nabla u)=\nabla^{\perp} A \cdot \nabla u .
\end{array}\right.
$$

Let $p \in B_{\frac{1}{2}}$ and $0<\rho<\frac{1}{2}$, we proceed by introducing on $B_{\rho}(p)$ the linear Hodge decomposition in $L^{2}$ of $A \nabla u$. Namely, there exist two functions $C$ and $D$, unique up to additive constants, elements of $W_{0}^{1,2}\left(B_{\rho}(p)\right)$ and $W^{1,2}\left(B_{\rho}(p)\right)$ respectively, and such that

$$
A \nabla u=\nabla C+\nabla^{\perp} D
$$

with

$$
\Delta C=\operatorname{div}(A \nabla u)=\nabla^{\perp} B \cdot \nabla u
$$

and

$$
\Delta D=-\nabla A \cdot \nabla^{\perp} u
$$

Wente's lemma 1.1 guarantees that $C$ lies in $W^{1,2}$, and moreover

$$
\int_{B_{\rho}(p)}|\nabla C|^{2} d z \leq C\left(\int_{B_{\rho}(p)}|\nabla B|^{2} d z\right)\left(\int_{B_{\rho}(p)}|\nabla u|^{2} d z\right)
$$


Then, we introduce the decomposition $D=\phi+v$, with $\phi$ satisfying

$$
\left\{\begin{array}{ccc}
\Delta \phi & =-\nabla A \cdot \nabla^{\perp} u & \text { in } \quad B_{\rho}(p) \\
\phi=0 & \text { on } \quad \partial B_{\rho}(p)
\end{array}\right.
$$

and with $v$ being harmonic. Once again, Wente's lemma 1.1 gives us the estimate

$$
\int_{B_{\rho}(p)}|\nabla \phi|^{2} d z \leq C\left(\int_{B_{\rho}(p)}|\nabla A|^{2} d z\right)\left(\int_{B_{\rho}(p)}|\nabla u|^{2} d z\right) .
$$

Using the fact that $\rho \mapsto \frac{1}{\rho^{2}} \int_{B_{\rho}(p)}|\nabla v|^{2} d z$ for any harmonic function, see lemma II.1 of [Ri6]. We get, for any $0 \leq \delta \leq 1$, that

$$
\int_{B_{\delta \rho}(p)}|\nabla v|^{2} d z \leq \delta^{2} \int_{B_{\rho}(p)}|\nabla v|^{2} d z
$$

Finally, we have

$$
\begin{aligned}
\int_{B_{\delta \rho}(p)}|\nabla D|^{2} d z & \leq 2 \delta^{2} \int_{B_{\rho}(p)}|\nabla D|^{2} d z \\
& +2 \int_{B_{\rho}(p)}|\nabla \phi|^{2} d z
\end{aligned}
$$

Bringing altogether (45), (46), and (48) produces

$$
\begin{aligned}
\int_{B_{\delta \rho}(p)}|A \nabla u|^{2} d z \leq & 2 \delta^{2} \int_{B_{\rho}(p)}|A \nabla u|^{2} d z \\
& +C \varepsilon_{0} \int_{B_{\rho}(p)}|\nabla u|^{2} d z .
\end{aligned}
$$

Using the hypotheses that $A$ and $A^{-1}$ are bounded in $L^{\infty}$, it follows from (49) that for all $0<\delta<1$, there holds the estimate

$$
\begin{aligned}
\int_{B_{\delta \rho}(p)}|\nabla u|^{2} d z \leq & 2\left\|A^{-1}\right\|_{\infty}\|A\|_{\infty} \delta^{2} \int_{B_{\rho}(p)}|\nabla u|^{2} d z \\
& +C\left\|A^{-1}\right\|_{\infty} \varepsilon_{0} \int_{B_{\rho}(p)}|\nabla u|^{2} d z .
\end{aligned}
$$

Next, we choose $\varepsilon_{0}$ and $\delta$ strictly positive, independent of $\rho$ et $p$, and such that

$$
2\left\|A^{-1}\right\|_{\infty}\|A\|_{\infty} \delta^{2}+C\left\|A^{-1}\right\|_{\infty} \varepsilon_{0}=\frac{1}{2}
$$

For this particular choice of $\delta$, we have thus obtained the inequality

$$
\int_{B_{\delta \rho}(p)}|\nabla u|^{2} d z \leq \frac{1}{2} \int_{B_{\rho}(p)}|\nabla u|^{2} d z .
$$

Classical results then yield the existence of some constant $\alpha>0$ for which

$$
\sup _{p \in B_{1 / 2}(0), 0<\rho<\frac{1}{2}} \rho^{-\alpha} \int_{B_{\rho}(p)}|\nabla u|^{2} d z<+\infty \quad,
$$


which prove the $\varepsilon$-regularity as already remark above.

Then, the energy gap follows easily remarking that, thanks to the conformal invariance, for all $R>0$ and some $q>2$, we have

$$
\|\nabla u\|_{L^{q}\left(B_{\frac{R}{4}}\right)} \leq \frac{C_{q}}{R^{\frac{q-2}{q}}}\|\nabla u\|_{L^{2}\left(B_{R}\right)} .
$$

Finally, the weak limit property is a just a special case of theorem I.5 of [Ri3] which is one of the many consequences of theorem 3.1

Then we are in position to prove theorem 0.2 which is the main result of this section once we will have established the following lemma.

Lemma 3.1. There exists $\delta>0$ such that for all $r, R \in \mathbb{R}_{+}^{*}$ satisfying $2 r<R$ for all $\Omega \in L^{2}\left(B_{R} \backslash\right.$ $\left.B_{r}, s o(n) \otimes \mathbb{R}^{n}\right)$ and $u \in W^{1,2}\left(B_{R} \backslash B_{r}, \mathbb{R}^{n}\right)$ satisfying

$$
-\Delta u=\Omega \cdot \nabla u
$$

and

$$
\sup _{r<\rho<\frac{R}{2}} \int_{B_{2 \rho} \backslash B_{\rho}}|\Omega|^{2} d z \leq \delta .
$$

Then there exists $C>0$, independent of $u, r$ and $R$, such that

$$
\|\nabla u\|_{L^{2, \infty}\left(B_{R} \backslash B_{r}\right)} \leq C\left[\sup _{r<\rho<\frac{R}{2}} \int_{B_{2 \rho} \backslash B_{\rho}}|\nabla u|^{2} d x\right]^{1 / 2} .
$$

Proof of lemma 3.1 .

Let

$$
\varepsilon:=\sup _{r<\rho<\frac{R}{2}} \int_{B_{2 \rho} \backslash B_{\rho}}|\nabla u|^{2} d z
$$

We assume $\delta$ to be smaller than $\varepsilon_{0}$ in the epsilon regularity result theorem 3.2 in such a way that for any $2 r<\rho<R / 4$ one has

$$
\left[\frac{1}{\rho^{2}} \int_{B_{2 \rho} \backslash B_{\rho}}|\nabla u|^{4}(x) d x\right]^{\frac{1}{4}} \leq C \frac{\sqrt{\varepsilon}}{\rho}
$$

Let $\lambda>0$. Let $f(x):=|\nabla u|$ in $B_{\frac{R}{2}} \backslash B_{2 r}$ and $f=0$ otherwise, we have that

$$
\forall \rho>0 \quad \int_{B_{2 \rho} \backslash B_{\rho}} f^{4}(x) d x \leq C \frac{\varepsilon^{2}}{\rho^{2}}
$$

For any $\rho>0$ denote

$$
U(\lambda, \rho):=\left\{x \in B_{2 \rho} \backslash B_{\rho} ; f(x)>\lambda\right\}
$$

With this notation, (53) implies that

$$
\lambda^{4}|U(\lambda, \rho)| \leq C \frac{\varepsilon^{2}}{\rho^{2}} .
$$

Let $k \in Z$ and $j \geq k$ we apply (54) for $\rho:=2^{j} \lambda^{-1}$ and by summing over $j \geq k$ one obtains

$$
\lambda^{2}\left|\left\{x \in \mathbb{R}^{2} \backslash B_{2^{k} \lambda^{-1}} ; f(x)>\lambda\right\}\right| \leq C \sum_{j=k}^{\infty} 2^{-2 j} \varepsilon^{2} \leq C 2^{-2 k} \varepsilon^{2}
$$


So we deduce that for any $k \in \mathbb{Z}$

$$
\lambda^{2}\left|\left\{x \in \mathbb{R}^{2} ; f(x)>\lambda\right\}\right| \leq C 2^{-2 k} \varepsilon^{2}+\pi 2^{2 k}
$$

Taking $2^{2 k} \simeq \varepsilon$ we obtain

$$
\|\nabla u\|_{L^{2, \infty}\left(B_{\frac{R}{2} \backslash B_{2 r}}\right)} \leq C\left[\sup _{r<\rho<\frac{R}{2}} \int_{B_{2 \rho} \backslash B_{\rho}}|\nabla u|^{2} d x\right]^{1 / 2} .
$$

using now the triangular inequality for the norm $L^{2, \infty}$ and the fact that the $L^{2, \infty}$ norm of $\nabla u$ is controled by the $L^{2}$ norm of $\nabla u$ over respectively $B_{R} \backslash B_{\frac{R}{2}}$ and $B_{2 r} \backslash B_{r}$, (56) implies (51) and lemma 3.1 is proved.

Proof of theorem 0.2:

Let $\varepsilon_{0}>0$ be the one of the theorem 3.1 .

\section{Step 1: We reduce the problem to an $L^{2,1}$ estimate}

Indeed, we use the duality $L^{2,1}-L^{2, \infty}$ in order to infer

$$
\int_{B_{\frac{R}{2} \backslash B_{2 r}}}\left|\frac{1}{\rho} \frac{\partial u}{\partial \theta}\right|^{2} d x \leq\left\|\frac{1}{\rho} \frac{\partial u}{\partial \theta}\right\|_{L^{2,1}\left(B_{\frac{R}{2} \backslash B_{2 r}}\right)}\left\|\frac{1}{\rho} \frac{\partial u}{\partial \theta}\right\|_{L^{2, \infty}\left(B_{\frac{R}{2} \backslash B_{2 r}}\right)}
$$

Combining this inequality with (51) we obtain

$$
\int_{B_{\frac{R}{2} \backslash B_{2 \rho}}}\left|\frac{1}{\rho} \frac{\partial u}{\partial \theta}\right|^{2} d x \leq C\left\|\frac{1}{\rho} \frac{\partial u}{\partial \theta}\right\|_{L^{2,1}\left(B_{\frac{R}{2} \backslash B_{2 r}}\right)}\left[\sup _{r<\rho<\frac{R}{2}} \int_{B_{2 \rho} \backslash B_{\rho}}|\nabla u|^{2} d x\right]^{1 / 2}
$$

Hence, thanks to duality, it suffices to control the $L^{2,1}$-norm of $\frac{1}{\rho} \frac{\partial u}{\partial \theta}$ by the $L^{2}$ norm of $\nabla u$ in the annulus in order to prove the theorem.

\section{Step 2: We prove the theorem assuming that}

$$
\int_{B_{R} \backslash B_{r}}|\Omega|^{2} d z<\varepsilon_{0}
$$

We start by extending $\Omega$, setting

$$
\widetilde{\Omega}=\left\{\begin{array}{l}
\Omega \text { on } B_{R} \backslash B_{r} \\
0 \text { on } B_{r}
\end{array}\right.
$$

Hence, thanks to theorem 3.1, there exists $\widetilde{A} \in W^{1,2}\left(B_{R}, G l_{n}(\mathbb{R})\right) \cap L^{\infty}\left(B_{R}, G l_{n}(\mathbb{R})\right)$ such that

$$
\operatorname{div}(\nabla \widetilde{A}-\widetilde{A} \widetilde{\Omega})=0
$$

and

$$
\int_{B_{R}}\left(|\nabla \widetilde{A}|^{2}+\left|\nabla \widetilde{A}^{-1}\right|^{2}\right) d z+\operatorname{dist}\left(\left\{\widetilde{A}, \widetilde{A}^{-1},\right\}, S O(n)\right) \leq C \int_{B_{R}}|\widetilde{\Omega}|^{2} d z
$$


Then, thanks to Poincaré's lemma, there exists $\widetilde{B} \in W^{1,2}\left(B_{R}(0), M_{n}(\mathbb{R})\right)$ such that

$$
\nabla \widetilde{A}-\widetilde{A} \widetilde{\Omega}=\nabla^{\perp} \widetilde{B}
$$

and, thanks to (58) and (59), we get

$$
\|\nabla \widetilde{B}\|_{L^{2}\left(B_{R}\right)} \leq C\|\Omega\|_{L^{2}\left(B_{R} \backslash B_{r}\right)},
$$

here $C$ is a constant independent of $\Omega$. Hence, u satisfies

$$
-\operatorname{div}(\widetilde{A} \nabla u)=\nabla^{\perp} \widetilde{B} \cdot \nabla u \text { on } B_{R} \backslash B_{r} .
$$

We extend $u$ to $B_{R}$ by $\widetilde{u}$ using the Whitney's extension theorem, see [AdFo] or [Ste for instance, then we get $\widetilde{u} \in W^{1,2}\left(B_{R}\right)$ such that

$$
\int_{B_{R}}|\nabla \widetilde{u}|^{2} d z \leq C \int_{B_{R} \backslash B_{r}}|\nabla u|^{2} d z
$$

We consider the Hodge decomposition of $\widetilde{A} \nabla \widetilde{u}$ on $B_{R}$, i.e there exists $C \in W_{0}^{1,2}\left(B_{R}\right)$ and $D \in W^{1,2}\left(B_{R}\right)$ such that

$$
\widetilde{A} \nabla \widetilde{u}=\nabla C+\nabla^{\perp} D .
$$

Moreover, thanks to (60), we get

$$
\int_{B_{R}}|\nabla C|^{2} d z+\int_{B_{R}}|\nabla D|^{2} d z=\int_{B_{R}}|\widetilde{A} \nabla \widetilde{u}|^{2} d z \leq C \int_{B_{R} \backslash B_{r}}|\nabla u|^{2} d z
$$

Here we use the fact that $C$ vanishes on the boundary to get that

$$
\int_{B_{R}} \nabla C \cdot \nabla^{\perp} D d z=0
$$

Then, on $B_{R} \backslash B_{r}, C$ satisfies

$$
\Delta C=\nabla^{\perp} \widetilde{B} \cdot \nabla u .
$$

As usual, we split as follows $C=v+\phi$ where $\phi \in W_{0}^{1,2}\left(B_{R} \backslash B_{r}\right)$ and $v \in W^{1,2}\left(B_{R} \backslash B_{r}\right)$ which satisfy

$$
\Delta \phi=\nabla^{\perp} \widetilde{B} \cdot \nabla u
$$

and

$$
\Delta v=0 .
$$

On the one hand, thanks to lemma 2.1 we get, for $0<\lambda<1$, that

$$
\|\nabla \phi\|_{L^{2,1}\left(B_{R} \backslash B_{\lambda^{-1}}\right)} \leq C(\lambda)\|\nabla \widetilde{B}\|_{2}\|\nabla u\|_{2}
$$

On the other hand, we decompose $v$ as a Fourier series,

$$
v=c_{0}+d_{0} \log (\rho)+\sum_{n \in \mathbb{Z}^{*}}\left(c_{n} \rho^{n}+d_{n} \rho^{-n}\right) e^{i n \theta} .
$$

since $\frac{1}{\rho} \frac{\partial v}{\partial \theta}$ has no logarithm part, we conclude as in lemma A.2 that for any $0<\lambda<1$ we have

$$
\left\|\frac{1}{\rho} \frac{\partial v}{\partial \theta}\right\|_{L^{2,1}\left(B_{\lambda R} \backslash B_{\left.\lambda^{-1} 1_{r}\right)}\right.} \leq C(\lambda)\|\nabla v\|_{2}
$$


The Dirichlet principle implies that

$$
\|\nabla v\|_{2} \leq\|\nabla C\|_{2}
$$

then we get

$$
\left\|\frac{1}{\rho} \frac{\partial C}{\partial \theta}\right\|_{L^{2,1}\left(B_{\lambda R} \backslash B_{\lambda^{-1} 1_{r}}\right)} \leq C(\lambda)\|\nabla u\|_{L^{2}\left(B_{R} \backslash B_{r}\right)} .
$$

Now we estimate $D$, which satisfies the following equation

$$
\Delta D=\nabla \widetilde{A} \cdot \nabla^{\perp} \widetilde{u} \text { on } B_{R} .
$$

Then, we also decompose $D$ as $D=v+\phi$ where $\phi \in W_{0}^{1,2}\left(B_{R}\right)$ and $v \in W^{1,2}\left(B_{R}\right)$.

$$
\Delta \phi=\nabla \widetilde{A} \cdot \nabla^{\perp} u
$$

and

$$
\Delta v=0
$$

In the one hand, thanks to lemma 1.1, we have

$$
\begin{aligned}
\|\nabla \phi\|_{2} & \leq\|\nabla \phi\|_{L^{2,1}\left(B_{R}\right)} \leq C\|\nabla \widetilde{A}\|_{2}\|\nabla \widetilde{u}\|_{2} \\
& \leq C\|\nabla u\|_{L^{2}\left(B_{R} \backslash B_{r}\right)} .
\end{aligned}
$$

in the other hand, since $v$ is harmonic, for any $0<\lambda<1$, we have

$$
\begin{aligned}
\|\nabla v\|_{L^{2,1}\left(B_{\lambda R}\right)} & \leq C(\lambda)\|\nabla v\|_{L^{2}\left(B_{R}\right)} \\
& \leq C(\lambda)\|\nabla D\|_{L^{2}\left(B_{R}\right)} \\
& \leq C(\lambda)\|\nabla u\|_{2} .
\end{aligned}
$$

Finally

$$
\|\nabla D\|_{L^{2,1}\left(B_{\lambda R} \backslash B_{\lambda^{-1} 1_{r}}\right)} \leq C(\lambda)\|\nabla u\|_{2} .
$$

Combining (61), (62) and (63), we get

$$
\left\|\widetilde{A} \frac{1}{r} \frac{\partial \widetilde{u}}{\partial \theta}\right\|_{L^{2,1}\left(B_{\lambda R} \backslash B_{\left.\lambda^{-1} 1_{r}\right)}\right.} \leq C(\lambda)\|\nabla u\|_{2} .
$$

Finally, using (58), we get that

$$
\left\|\frac{1}{\rho} \frac{\partial \widetilde{u}}{\partial \theta}\right\|_{L^{2,1}\left(B_{\lambda R} \backslash B_{\lambda}-1_{r}\right)} \leq C(\lambda)\|\nabla u\|_{2},
$$

which proves, as remark at the hand of step 1, the theorem under the extra assumption.

\section{Step 3: General case}

We construct two sequences of radii $r_{i}$ and $R_{i}$ such that

$$
r=r_{0}<r_{1}=R_{0}<\cdots<r_{i+1}=R_{i}<\cdots<R_{N}=R
$$


with

and

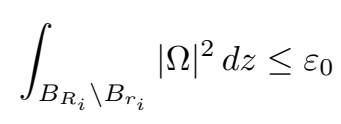

$$
N \leq \frac{\int_{B_{R} \backslash B_{r}}|\Omega|^{2} d z}{\varepsilon_{0}} .
$$

First, applying (64) of step 2, we get that

$$
\left\|\frac{1}{\rho} \frac{\partial u}{\partial \theta}\right\|_{L^{2,1}\left(B_{\lambda R_{i}} \backslash B_{\lambda-1}\right)} \leq C(\lambda)\|\nabla u\|_{L^{2}\left(B_{R_{i}} \backslash B_{r_{i}}\right)}
$$

We chose $\delta$ such that

$$
\delta<\frac{\varepsilon_{0}}{4}
$$

hence for all $i$ we have

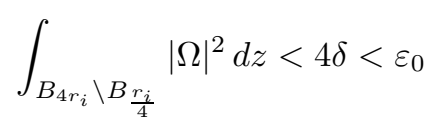

Let $S_{i}=\min \left(R, 4 r_{i}\right)$ and $s_{i}=\max \left(r, \frac{r_{i}}{4}\right)$, then we apply again (64) of step 2 on $B_{S_{i}} \backslash B_{s_{i}}$, which gives

$$
\left\|\frac{1}{\rho} \frac{\partial u}{\partial \theta}\right\|_{L^{2,1}\left(B_{\lambda S_{i}} \backslash B_{\lambda-1}\right)} \leq C(\lambda)\|\nabla u\|_{L^{2}\left(B_{S_{i}} \backslash B_{s_{i}}\right)}
$$

Finally, summing (65) and (66), for $i=0$ to $N$, we get

$$
\left\|\frac{1}{\rho} \frac{\partial u}{\partial \theta}\right\|_{L^{2,1}\left(B_{\lambda R} \backslash B_{\left.\lambda^{-1} 1_{r}\right)}\right.} \leq C(\lambda)\|\nabla u\|_{2},
$$

which achieves the proof of theorem 0.2 .

We shall now make use of the theorem 0.2 in order to prove the quantization of the angular part of the energy for solutions to antisymmetric elliptic systems.

We wil call a bubble a solution $u \in W^{2,1}\left(\mathbb{R}^{2}, \mathbb{R}^{n}\right)$ of the equation

$$
-\Delta u=\Omega \cdot \nabla u \text { on } \mathbb{R}^{2},
$$

where $\Omega \in L^{2}\left(\mathbb{R}^{2}\right.$, so $\left.(n) \otimes \mathbb{R}^{2}\right)$.

Proof of theorem 0.1 :

First we are going to separate $B_{1}$ in three parts: one where $u_{k}$ converge to a limit solution, some neighborhoods where the energy concentrates and where blow some bubbles and some neck regions which join the first two parts. This "bubble-tree" decomposition is by now classical, see $\mathrm{Pa}$ for instance, hence we just sketch briefly how to proceed.

\section{Step 1 : Find the point of concentration}

Let $\varepsilon_{0}$ be the one of theorem 3.2 and $\delta$ the one of theorem 0.2 . Then, thanks to (7), we easily proved that there exists finitely many points $a^{1}, \ldots, a^{n}$ where

$$
\int_{B\left(a_{i}, r\right)}\left|\Omega_{k}\right|^{2} d z \geq \varepsilon_{0} \text { for all } r>0 .
$$


Moreover, using theorem 3.2 , we prove that there exists $\Omega_{\infty} \in L^{2}\left(B_{1}, s o(n) \otimes \mathbb{R}^{2}\right)$ and $u_{\infty} \in W^{2,1}\left(B_{1}, \mathbb{R}^{n}\right)$ a solution of $-\Delta u=\Omega_{\infty} \cdot \nabla u$ on $B_{1}$, such that, up to a subsequence,

$$
\Omega_{k} \rightarrow \Omega_{\infty} \text { in } L_{l o c}^{2}\left(B_{1}, s o(n) \otimes \mathbb{R}^{2}\right),
$$

and

$$
u_{k} \rightarrow u_{\infty} \text { on } W_{l o c}^{1, p}\left(B_{1} \backslash\left\{a^{1}, \ldots, a^{n}\right\}\right) \text { for all } p \geq 1 .
$$

Of course, if $\left\|\Omega_{k}\right\|_{\infty}=O(1)$ or $\Omega_{k}=O\left(\nabla u_{k}\right)$, then $u_{k}$ is bounded in $W^{2, \infty}$ which gives the convergence in $C_{l o c}^{1, \eta}$ for all $\eta \in[0,1[$.

\section{Step 2 : Blow-up around $a^{i}$}

We choose $r_{i}>0$ such that

$$
\int_{B\left(a^{i}, r^{i}\right)}\left|\Omega_{\infty}\right|^{2} d z \leq \frac{\varepsilon_{0}}{4} .
$$

Then, we define a center of mass of $B\left(a^{i}, r^{i}\right)$ with respect to $\Omega_{k}$ in the following way

$$
a_{k}^{i}=\left(\frac{\int_{B\left(a^{i}, r^{i}\right)} x^{\alpha}\left|\Omega_{k}\right|^{2} d z}{\int_{B\left(a^{i}, r^{i}\right)}\left|\Omega_{k}\right|^{2} d z}\right)_{\alpha=1,2} .
$$

Let $\lambda_{k}^{i}$ be a positive real such that

$$
\int_{B\left(a_{k}^{i}, r^{i}\right) \backslash B\left(a_{k}^{i}, \lambda_{k}^{i}\right)}\left|\Omega_{k}\right|^{2} d z=\min \left(\delta, \frac{\varepsilon_{0}}{2}\right) .
$$

We set $\widetilde{u}_{k}(z)=u_{k}\left(a_{k}^{i}+\lambda_{k}^{i} z\right), \widetilde{\Omega}_{k}(z)=\lambda_{k}^{i} \Omega_{k}\left(a_{k}^{i}+\lambda_{k}^{i} z\right)$ and $N_{k}^{i}=B\left(a_{k}^{i}, r^{i}\right) \backslash B\left(a_{k}^{i}, \lambda_{k}^{i}\right)$.

Observe that the scaling we chose for defining $\widetilde{\Omega}_{k}(z)$ guaranties that

$$
\int_{B\left(0, r^{i} / \lambda_{k}^{i}\right)}\left|\widetilde{\Omega}_{k}\right|^{2} d z \leq \int_{B\left(a_{k}^{i}, r^{i}\right)}\left|\Omega_{k}\right|^{2} d x \leq C<+\infty
$$

moreover we have

$$
-\Delta \widetilde{u}_{k}=\widetilde{\Omega}_{k} \cdot \nabla \widetilde{u}_{k}
$$

Modulo extraction of a subsequence, we can assume that for each $i$

$$
\nabla \widetilde{u}_{k} \rightarrow \nabla \widetilde{u}_{\infty} \quad \text { weakly in } L_{l o c}^{2}\left(\mathbb{R}^{2}, \mathbb{R}^{n}\right) \quad \widetilde{\Omega}_{k} \rightarrow \widetilde{\Omega}_{\infty} \text { weakly in } L_{l o c}^{2}\left(\mathbb{R}^{2}, s o(n) \otimes \mathbb{R}^{2}\right)
$$

The weak limit property of theorem 3.2 implies that $\widetilde{u}_{\infty}$ and $\widetilde{\Omega}_{\infty}$ satisfy what we call a bubble equation

$$
-\Delta \widetilde{u}_{\infty}=\widetilde{\Omega}_{\infty} \cdot \nabla \widetilde{u}_{\infty} .
$$

\section{Step 3 : Iteration}

Two cases have to be considered separatly:

Either $\widetilde{\Omega}_{k}$ is subject to some concentration phenomena as (67), and then we find some new points of concentration and a limiting solution which is a bubble (possibly trivial), in other words a solution of $-\Delta u=\widetilde{\Omega}_{\infty} \cdot \nabla u$, where $\widetilde{\Omega}_{\infty}$ is a weak limit of $\widetilde{\Omega}_{k}$. In such a case we apply step 2 to our new concentration 
points.

Or, $\widetilde{u}_{k}$ converges uniformly on every compact subset to a bubble (possibly trivial).

Of course this process has to stop, since we are assuming a uniform bound on $\left\|\Omega_{k}\right\|_{2}$ and each step is consuming at least $\min \left(\delta, \frac{\varepsilon_{0}}{2}\right)$ of energy of $\Omega_{k}$. This process is sketched in the following picture.

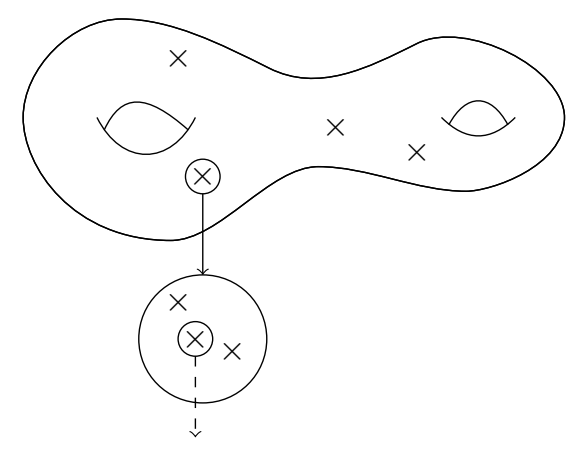

Figure 1: Decomposition of $\Sigma$

\section{Analysis of a neck region:}

A neck region is an annullar region $N_{k}^{i}=B\left(a_{k}^{i}, \mu_{k}^{i}\right) \backslash B\left(a_{k}^{i}, \lambda_{k}^{i}\right)$ such that

$$
\begin{gathered}
\lim _{k \rightarrow+\infty} \frac{\lambda_{k}^{i}}{\mu_{k}^{i}}=0, \\
\int_{N_{k}^{i}}\left|\Omega_{k}\right|^{2} d z \leq \min \left(\delta, \frac{\varepsilon_{0}}{2}\right)
\end{gathered}
$$

and

$$
X_{k}=\nabla^{\perp} d\left(a_{k}^{i}, .\right) .
$$

In order to prove theorem 0.1 , we start by proving a weak estimate on the energy of gradient in the neck region. First we remark that, for all $\varepsilon>0$, there exists $r>0$ such that for all $\rho>0$ such that

$$
B_{2 \rho}\left(a_{k}^{i}\right) \backslash B_{\rho}\left(a_{k}^{i}\right) \subset N_{k}^{i}(r)
$$

where $N_{k}^{i}(r)=B\left(a_{k}^{i}, r \mu_{k}^{i}\right) \backslash B\left(a_{k}^{i}, \frac{\lambda_{k}^{i}}{r}\right)$, we have

$$
\int_{B_{2 \rho}\left(a_{k}^{i}\right) \backslash B_{\rho}\left(a_{k}^{i}\right)}|\nabla u|^{2} d z \leq \varepsilon
$$

If this would not be the case there would exist a sequence $\rho_{k}^{i} \rightarrow 0$ such that, up to a subsequence, $\hat{u}_{k}=u_{k}\left(a_{k}^{i}+\rho_{k}^{i} z\right)$ converge with respect to every $W^{1, p}-$ norm to a non-trivial solution of

$$
-\Delta \hat{u}=\widehat{\Omega}_{\infty} \cdot \nabla \hat{u} \text { on } \mathbb{R}^{2} \backslash\{0\},
$$

where $\widehat{\Omega}_{\infty}$ is a weak limit, up to a subsequence, of $\widehat{\Omega}_{k}$. Using the fact that the $W^{1,2}$-norm of $\hat{u}_{k}$ is bounded, we deduce using Schwartz lemma that it has to be in fact a solution on the whole plane. Using 
this time the second part of theorem 3.2 we deduce that $\hat{\Omega}_{\infty}$ have energy at least $\varepsilon_{0}$, which contradicts (68).

Finally, using theorem 0.2 on each $N_{k}^{i}(r)$, we obtain

$$
\begin{aligned}
\lim _{r \rightarrow 0} \lim _{k \rightarrow+\infty}\left\|\left\langle\nabla u_{k}, X_{k}\right\rangle\right\|_{L^{2}\left(N_{k}^{i}(r)\right)} & \leq C \lim _{r \rightarrow 0} \lim _{k \rightarrow+\infty}\left(\sup _{\rho} \int_{B_{2 \rho}\left(a_{k}^{i}\right) \backslash B_{\rho}\left(a_{k}^{i}\right)}|\nabla u|^{2} d z\right) \\
& =0 .
\end{aligned}
$$

Which achieves the proof of theorem 0.1 .

This phenomena of quantization of the angular part of the gradient seems to be quite general for systems with antisymmetric potentials, in a forthcoming paper [LaRi] we investigate the quantization for some fourth order elliptic systems in 4-dimension.

\section{Energy Quantization for critical points to conformally invari- ant lagrangians.}

In the present section we are going to use theorem 0.1 in order to prove theorem 0.3

In his proof of the Heinz-Hildebrandt's regularity conjecture, the second author prove that the Euler Lagrange equations to general conformally invariant lagrangians which are coercive and of quadratic growth can be written in the form of an elliptic system with an antisymmetric potential. Precisely we have

Theorem 4.1 (Theorem I.2 [Ri3]). Let $N^{k}$ be a $C^{2}$ submanifold of $\mathbb{R}^{m}$ and $\omega$ be a $C^{1} 2$-form on $N^{k}$ such that the $L^{\infty}$-norm of $d \omega$ is bounded on $N^{k}$. Then every critical point in $W^{1,2}\left(B_{1}, N^{k}\right)$ of the Lagrangian

$$
F(u)=\int_{B_{1}}\left[|\nabla u|^{2}+u^{*} \omega\right] d z
$$

satisfies

$$
-\Delta u=\Omega \cdot \nabla u
$$

with

$$
\Omega_{j}^{i}=\left[A^{i}(u)_{j, l}-A^{j}(u)_{i, l}\right] \nabla u^{l}+\frac{1}{4}\left[H^{i}(u)_{j, l}-H^{j}(u)_{i, l}\right] \nabla^{\perp} u^{l}
$$

where $A$ and $\lambda$ are in $C^{0}\left(B_{1}, M_{m}(\mathbb{R}) \otimes \bigwedge^{1} \mathbb{R}^{2}\right)$ satisfy

$$
\sum_{j=1}^{m} A_{i, l}^{j} \nabla u^{j}=0
$$

and $H_{j, l}^{i}:=d\left(\pi^{*} \omega\right)\left(\varepsilon_{i}, \varepsilon_{j}, \varepsilon_{l}\right)$ where, in a neighborhood of $N^{k}, \pi$ is the orthogonal projection onto $N^{k}$ and $\left(\varepsilon_{i}\right)_{i=1 \cdots m}$ is the canonical basis of $\mathbb{R}^{m}$.

From (71) we observe that for critical points to a conformally invariant $C^{1}$-Lagrangian, there exists

$$
\Lambda \in C^{0}\left(T N \otimes \mathbb{R}^{2}, s o(n) \otimes \mathbb{R}^{2}\right)
$$

such that

$$
\Lambda(v)=O(|v|)
$$


moreover we remark that $\Lambda(u, \nabla u) \cdot \nabla u$ is always orthogonal to $\nabla u$ in the following sense

$$
\left\langle\frac{\partial u}{\partial x_{k}}, \Lambda(u, \nabla u) \cdot \nabla u\right\rangle=0 \text { for } k=1,2 .
$$

For $\Lambda \in C^{0}\left(T N \otimes \mathbb{R}^{2}, s o(n) \otimes \mathbb{R}^{2}\right)$, we call a $\Lambda$-bubble a solution $\omega \in W^{2,1}\left(\mathbb{R}^{2}, \mathbb{R}^{n}\right)$ of the equation

$$
-\Delta \omega=\Lambda(\omega, \nabla \omega) \cdot \nabla \omega \text { on } \mathbb{R}^{2} .
$$

Theorem 4.2. Let $u_{k} \in W^{1,2}\left(B_{1}, \mathbb{R}^{n}\right)$ be a sequence of critical points of a functional which is conformally invariant, which satisfies

$$
-\Delta u_{k}=\Lambda\left(u_{k}, \nabla u_{k}\right) \cdot \nabla u_{k},
$$

where $\Lambda$ satisfies (72), (73) and (74). Moreover we assume that $u_{k}$ has a bounded energy, i.e.

$$
\left\|\nabla u_{k}\right\|_{2} \leq M
$$

Then there exists $u_{\infty} \in W^{1,2}\left(B_{1}, \mathbb{R}^{n}\right)$ a solution of $-\Delta u_{\infty}=\Lambda\left(u_{\infty}, \nabla u_{\infty}\right) \cdot \nabla u_{\infty}$ on $B_{1}, l \in \mathbb{N}^{*}$ and

1. $\omega^{1}, \ldots, \omega^{l}$ some non-constant $\Lambda$-bubbles

2. $a_{k}^{1}, \ldots, a_{k}^{l}$ a family of converging sequences of points of $B_{1}$

3. $\lambda_{k}^{1}, \ldots, \lambda_{k}^{l}$ a family of sequences of positive reals converging all to zero.

such that, up to a subsequence,

$$
u_{k} \rightarrow u^{\infty} \text { on } C_{l o c}^{1, \eta}\left(B_{1} \backslash\left\{a_{\infty}^{1}, \ldots, a_{\infty}^{l}\right\}\right) \text { for all } \eta \in[0,1[
$$

and

$$
\left\|\nabla\left(u_{k}-u_{\infty}-\sum_{i=1}^{l} \omega_{k}^{i}\right)\right\|_{L_{l o c}^{2}\left(B_{1}\right)} \rightarrow 0
$$

where $\omega_{k}^{i}=\omega\left(a_{k}^{i}+\lambda_{k}^{i} z\right)$.

Since (74) holds for any system issued from a lagrangian of the form (70), it is clear that theorem 0.3 is a consequence of theorem 4.2

Proof of theorem 4.2 :

From the previous section, we have the quantization of the angular part of the gradient. To prove theorem 4.2 it suffices then to prove the energy quantization for the radial part of the energy. Since $u_{k}$ satisfies (75) then $u_{k} \in W^{2, p}\left(B_{\mu_{k}^{i}}\left(a_{k}^{i}\right)\right)$ for all $p<\infty$, see theorem IV.3 of [Ri6] or lemma 7.1 of [ShTo], hence we can multiply (75) by $\rho \frac{\partial u_{k}}{\partial \rho}$ and integrate. Using (174) we have, for any $r \in\left[0, \mu_{k}^{i}\right]$,

$$
0=\int_{B_{r}}\left\langle\rho \frac{\partial u_{k}}{\partial \rho}, \Omega \cdot \nabla u_{k}\right\rangle d z=\int_{B_{r}}\left\langle\rho \frac{\partial u_{k}}{\partial \rho}, \Delta u_{k}\right\rangle d z
$$

Using Pohozaev identity, we get for all $r \in\left[0, \mu_{k}^{i}\right]$

$$
\int_{\partial B_{r}}\left|\frac{\partial u_{k}}{\partial \rho}\right|^{2} d \sigma=\int_{\partial B_{r}}\left|\frac{1}{\rho} \frac{\partial u_{k}}{\partial \theta}\right|^{2} d \sigma
$$


Finally, we have

$$
\lim _{r \rightarrow 0} \lim _{k \rightarrow+\infty}\left\|\nabla u_{k}\right\|_{L^{2}\left(N_{k}^{i}(r)\right)}=0
$$

which concludes the proof of the theorem.

In particular we get the quantization for the solution of the problem of prescribed mean curvature. Indeed, an immersion of a Riemann surface $\Sigma$ into $\mathbb{R}^{3}$ with prescribed mean curvature $H \in C^{0}\left(\mathbb{R}^{3}, \mathbb{R}\right)$ satisfies the following $H$-system

$$
\Delta u=2 H(u) u_{x} \wedge u_{y},
$$

where $z=x+i y$ are some local conformal coordinates on $\Sigma$.

In order to state precisely our theorem, we define the notion of $H$-bubble as being a map $\omega \in$ $W^{1,2}\left(\mathbb{R}^{2}, \mathbb{R}^{3}\right)$ satisfying

$$
\Delta \omega=2 H(\omega) \omega_{x} \wedge \omega_{y} \text { on } \mathbb{R}^{2} .
$$

We shall also rescale the Riemann surface around a point. To that aim we will introduce some conformal chart. Precisely there exists $\delta>0$ such that for any $a \in \Sigma$ and $0<\lambda<\delta$ there exists a map $\Phi_{a, \lambda}$ : $B(a, \delta) \rightarrow \mathbb{R}^{2}$ which is a conformal-diffeomorphism, sends $a$ to 0 and $B(a, \lambda)$ to $B(0,1)$. We also associate to each point a cut-off function $\chi_{a} \in C^{\infty}(\Sigma)$ which satisfies

$$
\left\{\begin{array}{l}
\chi_{a} \equiv 1 \text { on } B\left(a, \frac{\delta}{2}\right) \\
\chi_{a} \equiv 0 \text { on } \Sigma \backslash B(a, \delta) .
\end{array}\right.
$$

Corollary 4.1. Let $\Sigma$ be a closed Riemann surface, $H \in C^{0}\left(\mathbb{R}^{3}, \mathbb{R}\right)$ and $u_{k} \in W^{2,1}\left(\Sigma, \mathbb{R}^{3}\right)$ a sequence of non-constant solution of (76) on $\Sigma$ then there exists, $u_{\infty} \in W^{2,1}\left(\Sigma, \mathbb{R}^{3}\right)$ a solution of (76), $k \in \mathbb{N}^{*}$ and

1. $\omega^{1}, \ldots, \omega^{l}$ a family of $H$-bubbles

2. $a_{k}^{1}, \ldots, a_{k}^{l}$ a family of converging sequences of point of $\Sigma$

3. $\lambda_{k}^{1}, \ldots, \lambda_{k}^{l}$ a family of sequences of positive reals converging all to zero

$$
u_{k} \rightarrow u^{\infty} \text { on } C_{l o c}^{1, \eta}\left(\Sigma \backslash\left\{a_{1}^{\infty}, \ldots, a_{k}^{\infty}\right\}\right) \text { for all } \eta \in[0,1[
$$

and moreover

$$
\left\|\nabla\left(u_{k}-u_{\infty}-\sum_{i=1}^{l} \chi_{a_{k}^{i}}\left(\omega^{i} \circ \Phi_{a_{k}^{i}, \lambda_{k}^{i}}\right)\right)\right\|_{2} \rightarrow 0 .
$$

We end up this section by mentioning a recent work by Da Lio, DaL in which energy quantization results for fractional harmonic maps (which are also conformally invariant in some dimension) are established using also Lorentz space uniform estimates.

\section{Other applications to pseudo-holomorphic curves, harmonic maps and Willmore surfaces}

In this section we give some more applications of the uniform Lorentz-Wente estimates of section 2 to problems where the conformal invariance play again a central role. 
In the present section we are interested with Wente's type estimate for first order system of the form

$$
\nabla \phi=\sum_{i=1}^{n} a_{i} \nabla^{\perp} b_{i}
$$

Taking the divergence of this system gives the classical order 2 Wente system

$$
\Delta \phi=\sum_{i=1}^{n} \nabla a_{i} \cdot \nabla^{\perp} b_{i}
$$

The gain of information provided by a first order system of the form (77) in comparison to classical second order system (78) is illustrated by the fact that, in the first order case, no assumption on the behavior of the solution $\phi$ at the boundary of the annulus is needed in order to obtain the Lorentz - Wente type estimates of section 2. This is proved in lemma 5.1. This fact can be applied to geometrically interesting situations that we will describe at the end of the present section.

\subsection{Lorentz-Wente type estimates for first order Wente type equations.}

The goal of this subsection is to prove the following lemma.

Lemma 5.1. Let $n \in \mathbb{N}^{*},\left(a_{i}\right)_{1 \leq i \leq n}$ and $\left(b_{i}\right)_{1 \leq i \leq n}$ be two families of maps in $W^{1,2}\left(B_{1}\right), 0<\varepsilon<\frac{1}{4}$ and $\phi \in W^{1,2}\left(B_{1} \backslash B_{\varepsilon}\right)$ which satisfies

$$
\nabla \phi=\sum_{i=1}^{n} a_{i} \nabla^{\perp} b_{i}
$$

Then, for $0<\lambda<1$, there exists a positive constant $C(\lambda)$ independent of $\phi, a_{i}$ and $b_{i}$ such that

$$
\|\nabla \phi\|_{L^{2,1}\left(B_{\lambda} \backslash B_{\lambda-1}\right)} \leq C(\lambda)\left(\sum_{i=1}^{n}\left\|\nabla a_{i}\right\|_{2}\left\|\nabla b_{i}\right\|_{2}+\|\nabla \phi\|_{2}\right) .
$$

Proof of lemma 5.1:

Taking the divergence of (79), gives

$$
\Delta \phi=\sum_{i=1}^{n} \nabla a_{i} \cdot \nabla^{\perp} b_{i}
$$

Hence, as in the previous lemma, we start by considering a solution of this equation on the whole disk and equal to zero on the boundary. Let $\varphi \in W_{0}^{1,1}\left(B_{1}\right)$ be the solution of

$$
\Delta \varphi=\sum_{i=1}^{n} \nabla a_{i} \cdot \nabla^{\perp} b_{i}
$$

Then, thanks to the improved Wente's inequality (10), we have

$$
\|\nabla \varphi\|_{L^{2,1}\left(B_{1}\right)} \leq C \sum_{i=1}^{n}\left\|\nabla a_{i}\right\|_{2}\left\|\nabla b_{i}\right\|_{2}
$$


We now consider the difference $v=\phi-\varphi$, which is an harmonic function on $B_{1} \backslash B_{\varepsilon}$. Following the proof of the lemma A.2, it suffices to control the logarithmic part of the decomposition in Fourier series. To that aim we set

$$
\bar{\phi}(\rho)=\frac{1}{2 \pi} \int_{0}^{2 \pi} \phi(\rho, \theta) d \theta
$$

We have

$$
\begin{aligned}
\frac{d \bar{\phi}}{d \rho} & =\frac{1}{2 \pi} \int_{0}^{2 \pi} \frac{\partial \phi}{\partial \rho}(\rho, \theta) d \theta=\frac{1}{2 \pi} \sum_{i=1}^{n} \int_{0}^{2 \pi} a_{i} \frac{\partial b_{i}}{\partial \theta} \frac{d \theta}{\rho} \\
& =\frac{1}{2 \pi} \sum_{i=1}^{n} \int_{0}^{2 \pi}\left(a_{i}-\overline{a_{i}}\right) \frac{\partial b_{i}}{\partial \theta} \frac{d \theta}{\rho}
\end{aligned}
$$

Hence

$$
\left|\frac{d \bar{\phi}}{d \rho}\right| \leq \frac{1}{2 \pi} \sum_{i=1}\left(\int_{0}^{2 \pi}\left|a_{i}-\overline{a_{i}}\right|^{2} d \theta\right)^{\frac{1}{2}}\left(\int_{0}^{2 \pi}\left|\frac{1}{\rho} \frac{\partial b_{i}}{\partial \theta}\right|^{2} d \theta\right)^{\frac{1}{2}}
$$

Which gives, thanks to Poincaré's inequality on the circle,

$$
\left|\frac{d \bar{\phi}}{d \rho}\right| \leq C \sum_{i=1}\left(\int_{0}^{2 \pi}\left|\frac{\partial a_{i}}{\partial \theta}\right|^{2} d \theta\right)^{\frac{1}{2}}\left(\int_{0}^{2 \pi}\left|\frac{1}{\rho} \frac{\partial b_{i}}{\partial \theta}\right|^{2} d \theta\right)^{\frac{1}{2}}
$$

where $C$ is a constant independent of $\phi$.

Then integrating over $[1, \varepsilon]$, we get

$$
\begin{aligned}
\int_{\varepsilon}^{1}\left|\frac{d \bar{\phi}}{d \rho}\right| d \rho & \leq C \sum_{i=1}^{n} \int_{\varepsilon}^{1}\left(\int_{0}^{2 \pi}\left|\frac{\partial a_{i}}{\partial \theta}\right|^{2} d \theta\right)^{\frac{1}{2}}\left(\int_{0}^{2 \pi}\left|\frac{1}{\rho} \frac{\partial b_{i}}{\partial \theta}\right|^{2} d \theta\right)^{\frac{1}{2}} d \rho \\
& \leq C \sum_{i=1}^{n}\left(\int_{\mathrm{D}(0,1) \backslash B_{\varepsilon}}\left|\frac{1}{\rho} \frac{\partial a_{i}}{\partial \theta}\right|^{2} \rho d \rho d \theta\right)^{\frac{1}{2}}\left(\int_{\mathrm{D}(0,1) \backslash B_{\varepsilon}}\left|\frac{1}{\rho} \frac{\partial b_{i}}{\partial \theta}\right|^{2} \rho d \rho d \theta\right)^{\frac{1}{2}} \\
& \leq C\left(\sum_{i=1}^{n}\left\|\nabla a_{i}\right\|_{2}\left\|\nabla b_{i}\right\|_{2}\right)
\end{aligned}
$$

Moreover, by duality, we obtain

$$
\int_{\varepsilon}^{1}\left|\frac{d \bar{\varphi}}{d \rho}\right| d \rho \leq\left\|\nabla \varphi \frac{1}{\rho}\right\|_{1} \leq\|\nabla \varphi\|_{L^{2,1}}\left\|\frac{1}{\rho}\right\|_{L^{2, \infty}} \leq C\|\nabla \varphi\|_{L^{2,1}} .
$$

The combination of (80), (81) and (82) gives then

$$
\int_{\varepsilon}^{1}\left|\frac{d \bar{v}}{d \rho}\right| d \rho \leq C\left(\sum_{i=1}^{n}\left\|\nabla a_{i}\right\|_{2}\left\|\nabla b_{i}\right\|_{2}\right)
$$

Following the approaches we used in the proofs of the various lemma in section 2 , we decompose $v$ as a Fourier series, which gives

$$
v(\rho, \theta)=c_{0}+d_{0} \ln (\rho)+\sum_{n \in \mathbb{Z}^{*}}\left(c_{n} \rho^{n}+d_{n} \rho^{-n}\right) e^{i n \theta}
$$


We have

$$
\bar{v}(\rho)=c_{0}+d_{0} \ln (\rho)
$$

Thanks to (83), we get that

$$
\left|d_{0}\right| \log \frac{1}{\varepsilon} \leq C\left(\sum_{i=1}^{n}\left\|\nabla a_{i}\right\|_{2}\left\|\nabla b_{i}\right\|_{2}\right)
$$

We have moreover

$$
\begin{array}{r}
\|\nabla \bar{v}\|_{L^{2,1}\left(B_{1} \backslash B_{\varepsilon}\right)} \simeq\left|d_{0}\right| \int_{0}^{\infty}\left|\left\{x \in B_{1} \backslash B_{\varepsilon} ;|x|^{-1}>t\right\}\right|^{1 / 2} d t \\
=\left|d_{0}\right| \int_{0}^{\infty}\left|\left(B_{1} \backslash B_{\varepsilon}\right) \cap B_{1 / t}\right|^{1 / 2} d t \leq \pi\left|d_{0}\right| \int_{0}^{1 / \varepsilon} \frac{d t}{\max \{t, 1\}}=\pi\left|d_{0}\right|\left[1+\log \frac{1}{\varepsilon}\right]
\end{array}
$$

Thus combining (84) and (85) we have in one hand

$$
\|\nabla \bar{v}\|_{L^{2,1}\left(B_{1} \backslash B_{\varepsilon}\right)} \leq C\left(\sum_{i=1}^{n}\left\|\nabla a_{i}\right\|_{2}\left\|\nabla b_{i}\right\|_{2}\right),
$$

in the other hand, as in lemma A.2, we have

$$
\left\|\sum_{n \in \mathbb{Z}^{*}}\left(c_{n} \rho^{n}+d_{n} \rho^{-n}\right) e^{i n \theta}\right\|_{L^{2,1}\left(B_{\lambda} \backslash B_{\lambda}-1_{\varepsilon}\right)} \leq C(\lambda)\|\nabla v\|_{2} \leq C(\lambda)\|\nabla \phi\|_{2} .
$$

Combining (86), (87) we have for any $\lambda \in(0,1)$ the existence of a positive constant $C(\lambda)>0$ such that

$$
\|\nabla v\|_{L^{2,1}\left(B_{\lambda} \backslash B_{\lambda-1}\right)} \leq C(\lambda)\left(\sum_{i=1}^{n}\left\|\nabla a_{i}\right\|_{2}\left\|\nabla b_{i}\right\|_{2}+\|\nabla \phi\|_{2}\right) .
$$

Finally summing (80) and (188) gives the desired inequality and lemma 5.1 is proved.

\subsection{Quantization of pseudo-holomorphic curves on degenerating Riemann surfaces}

We consider a closed Riemann surfaces $(\Sigma, h)$, where $\Sigma$ is smooth compact surface without boundary, and $h$ a metric on $\Sigma$. Since we are only interested in the conformal structure of $\Sigma$, we can assume, thanks to the uniformization theorem, see [Hub], that $h$ has constant scalar curvature. We consider $(N, J)$ to be a smooth almost-complex manifold and we look at pseudo-holomorphic curves between $(\Sigma, h)$ and $(N, J)$, in other words we consider applications $u \in W^{1,2}(\Sigma, N)$ satisfying

$$
\frac{\partial u}{\partial x}=J(u) \frac{\partial u}{\partial y}
$$

where $z=x+i y$ are some local conformal coordinates on $\Sigma$. These objects are fundamental in symplectic geometry, see [McDS]. In the study of the moduli space of pseudo-holomorphic curves in an almost complex manifold, the compactification question comes naturally. In other words it is of first importance to understand and describe how sequences of pseudo-holomorphic curves with possibly degenerating conformal class behave at the limit.

The so-called Gromov's compactness theorem [Gr], see also [PW], Si] and [Hum], provides an answer to this question. 
Theorem 5.1. [Gr] Let $(N, J)$ a compact almost manifold, $\Sigma$ a closed surface and $\left(j_{n}\right)$ a sequence of complex structures on $\Sigma$. Assume $u_{n}:\left(\Sigma, j_{n}\right) \rightarrow(N, J)$ is a sequence of pseudo-holomorphic curves of bounded area with respect to an arbitrary metric on $N$. Then $u_{n}$ converge weakly to some cusp curve 4 $\bar{u}: \bar{\Sigma} \rightarrow(N, J)$ and there exists finitely many bubbles, holomorphic maps $\left(\omega^{i}\right)_{i=1 \cdots l}$ from $S^{2}$ into $(N, J)$, such that, modulo extraction of a subsequence

$$
\lim _{n \rightarrow+\infty} E\left(u_{n}\right)=E(\bar{u})+\sum_{i=1}^{l} E\left(\omega^{i}\right)
$$

In fact the bound, on the energy is not necessary assuming that the target manifold is symplectic, i.e if there is $\omega$ a closed 2-form on $N$ compatible with $J$. In deed, in that case, see chapter 2 of [McDS] for instance, all $u: \Sigma \rightarrow N(J, \omega)$, regular enough, satisfies

$$
A(u)=\int_{\Sigma} d v o l_{u^{*} g} \geq \int_{\Sigma} u^{*} \omega
$$

where $g=\omega(., J$.$) , with equality if and only if u$ is pseudo-holomorphic. Hence, for symplectic manifold, pseudo-holomorphic curves are area minimizing in their homology class. In particular, they are minimal surfaces, i.e. conformal and harmonic, and we can use the general theory of harmonic maps, see remark 4.2 of $[\mathrm{Zhu}]$.

We propose below a proof of theorem 5.1 that follows the main lines of the most classical one (i.e. we shall decompose our curves in thin and thick parts at the limit) but the argument we provide in order to prove that there is no energy in the neck and collar regions is new. We don't make use of the standard isoperimetric machinery but we simply apply the first order Wente's estimate on annuli given by lemma 5.1 which fits in an optimal way the particular structure of the pseudo-holomorphic equation (89) .

\section{Proof of theorem 5.1:}

The proof consists in splitting the surface in several pieces where the sequence converges either strongly to a non-constant limiting map or weakly to a constant. Then in a second step, we prove that there is in fact no energy in the pieces where the converge is weak. Note that in contrast to the previous section, in the present case the complex structure of the surface is not fixed and is a priori free to degenerate.

$\mathrm{Ou}$ aim is to show how lemma 5.1 can be used in this context and therefore we shall be more brief on the classical parts such as the limiting Deligne-Mumford thin-thick decomposition which is described for instance in $\mathrm{Hum}$, or in [ $\mathrm{Zhu}$. Observe that. due to the structure of the equation the $\varepsilon$-regularity theorem for pseudo-holomorphic curves is a consequence of theorem 3.2 .

For simplicity, we will also assume that we have a surface of genus $g$ greater or equal to 2 . Hence let $h_{n}$ be the hyperbolic metric of volume 1 associated to the complex structure $j_{n}$,

According to the Deligne-Mumford compactification of Riemann surfaces, see chapter 4 of [Hum], modulo extraction of a subsequence, $\left(\Sigma, h_{n}\right)$ converges to an hyperbolic Riemannian $(\Sigma, h)$ surface by collapsing $p(0 \leq p \leq 3 g-3)$ pairwise disjoint simple closed geodesics $\left(\gamma_{n}^{i}\right)$.

Far from the collapsing geodesics, the metric uniformly converges, and we have a classical "bubbletree" decomposition, that is to say $u_{n}$ converges to a pseudo-holomorphic curves of the $(\Sigma, h)$ expect

\footnotetext{
${ }^{4}$ we refer to chapter 5 of [Hum] for precise definitions
} 
possibly at finitely many points where, as in the previous section, $u_{n}$ is forming bubbles (i.e pseudoholomorphic curves from $\mathbb{C}$ to $N$ ) which are "connected" to each other by some neck regions $N_{n}^{i}=$ $B\left(a_{n}^{i}, \mu_{n}^{i}\right) \backslash B\left(a_{n}^{i}, \lambda_{n}^{i}\right)$ where the weak $L^{2}$ energy goes to zero,

$$
\lim _{r \rightarrow 0} \lim _{n \rightarrow+\infty}\left\|\nabla u_{n}\right\|_{L^{2, \infty}\left(N_{n}^{i}(r)\right)}=0
$$

where $N_{n}^{i}(r)=B\left(a_{n}^{i}, r \mu_{n}^{i}\right) \backslash B\left(a_{n}^{i}, \frac{\lambda_{n}^{i}}{r}\right)$. This can be established by combining the fact that, on such annular regions, the maximal $L^{2}$ energy of $\nabla u_{n}$ on dyadic annuli has to vanish (otherwise we would have another bubble) and the fact that lemma 3.1 applies to this situation.

Near the collapsing geodesics, our surface becomes asymptotically isometric to an hyperbolic cylinder of the form

$$
A_{l}=\left\{z=r e^{i \phi} \in \mathbb{H}: 1 \leq r \leq e^{l}, \arctan \left(\sinh \left(\frac{l}{2}\right)\right)<\phi<\pi-\arctan \left(\sinh \left(\frac{l}{2}\right)\right)\right\}
$$

where the geodesic correspond to $\left\{r e^{i \frac{\pi}{2}} \in \mathbb{H}: 1 \leq r \leq e^{l}\right\}$ and the line $\{r=1\}$ and $\left\{r=e^{l}\right\}$ are identified via $z \mapsto e^{l} z$. This is the collar region. It is sometimes easier to consider the following cylindrical parametrization, i.e.

$$
P_{l}=\left\{(t, \theta): \frac{2 \pi}{l} \arctan \left(\sinh \left(\frac{l}{2}\right)\right)<t<\frac{2 \pi}{l}\left(\pi-\arctan \left(\sinh \left(\frac{l}{2}\right)\right)\right), 0 \leq \theta \leq 2 \pi\right\}
$$

in this parametrization the constant scalar curvature metric reads

$$
d s^{2}=\left(\frac{l}{2 \pi \sin \left(\frac{l t}{2 \pi}\right)}\right)^{2}\left(d t^{2}+d \theta^{2}\right)
$$

where the geodesic corresponds to $\left\{t=\frac{\pi^{2}}{l}\right\}$ and the line $\{\theta=0\}$ and $\{\theta=2 \pi\}$ are identified.

Then, as $l_{n}$, the length of the degenerating geodesic, goes to zero, $P_{l_{n}}=\left[0, T_{n}\right] \times S^{1}$ up to translation, which can be decompose as follows, see proposition 3.1 of [Zhu].

For each such a thin part, one can extracts a subsequence such that the following decomposition holds. There $p \in \mathbb{N}$ and exists $2 \mathrm{p}$ sequences $\left(a_{n}^{1}\right),\left(b_{n}^{1}\right),\left(a_{n}^{2}\right),\left(b_{n}^{2}\right), \ldots,\left(a_{n}^{p}\right),\left(b_{n}^{p}\right)$ of positive numbers between 0 and $T_{n}$ such that

$$
\lim _{n \rightarrow+\infty} \frac{b_{n}^{i}-a_{n}^{i}}{T_{n}}=0
$$

and up to rescaling and identifying $]-\infty,+\infty\left[\times S^{1}\right.$ with $\mathbb{C} \backslash\{0\}$, there exists a bubble $\omega^{i}$ (i.e pseudoholomorphic curve from $\mathbb{C}$ to $N$ ) such that

$$
u^{n}\left(\frac{a_{n}^{i}+b_{n}^{i}}{2}+\frac{t}{b_{n}^{i}-a_{n}^{i}}, \theta\right) \rightarrow \omega^{i} \text { on } C_{\text {loc }}^{2}(\mathbb{C} \backslash\{0\}) .
$$

Moreover, for any $\varepsilon>0$, there exists $r>0$ such that for any $T \in\left[b_{n}^{i}+r^{-1}, a_{n}^{i+1}-r^{-1}\right]$

$$
\int_{[T, T+1] \times S^{1}}\left|\nabla u_{n}\right|^{2} \leq \varepsilon
$$

Denoting $J_{n}^{i}=\left[a_{n}^{i}, b_{n}^{i}\right] \times S^{1}, I_{n}^{0}=\left[0, a_{n}^{1}\right] \times S^{1}, I_{n}^{i}=\left[b_{n}^{i}, a_{n}^{i+1}\right] \times S^{1}$ and $I_{n}^{p}=\left[b_{n}^{p}, T_{n}\right] \times S^{1}$ and $I_{n}^{i}(r)=$ $\left[b_{n}^{i}+r^{-1}, a_{n}^{i+1}-r^{-1}\right]$, (90) combined with lemma 3.1 implies

$$
\lim _{r \rightarrow 0} \lim _{n \rightarrow+\infty}\left\|\nabla u_{n}\right\|_{L^{2, \infty}\left(I_{n}^{i}(r)\right)}=0
$$




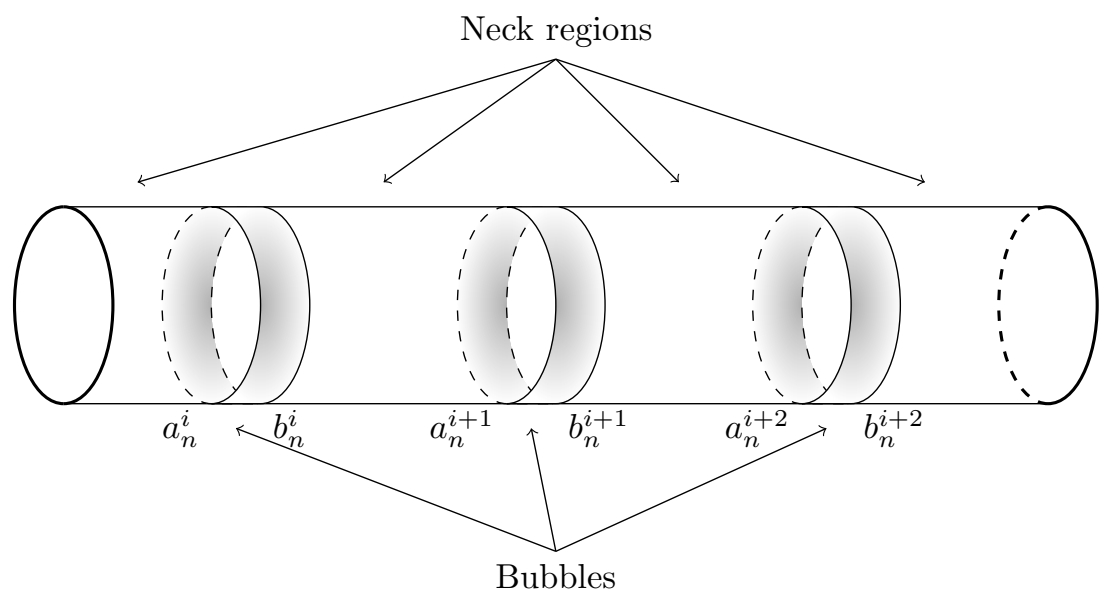

Figure 2: Decomposition in necks and bubbles

This decomposition is illustrated by the following picture.

As in the previous section, in order to prove that there is no energy at the limit in the neck regions of the thin parts, we combine the vanishing of the $L^{2, \infty}$-norm given by (91) with a uniform estimate on the $L^{2,1}$ norm of $\left|\nabla u^{n}\right|$ on each $I_{n}^{i}(r)$, which is a direct consequence of the lemma 5.1 applied to the pseudo-holomorphic equation

$$
\nabla u_{n}=J\left(u_{n}\right) \nabla^{\perp} u_{n}
$$

This concludes the proof of theorem 5.1

Remark 5.1. Here again, in addition to the fact that our argument is not specific to J-holomorphic curves, our proof, in comparison with previous ones such as the one given in [Zhu], has the advantage to require less regularity on the target manifold $N$. In fact, following the approach of [Pa] or [LiWa], in order to establish the angular energy quantization, M.Zhu goes through a lower estimate of the following second derivative

$$
\frac{d^{2}}{d \theta^{2}} \int_{S^{1} \times\{t\}}\left|u_{\theta}\right|^{2} d \theta
$$

Such an estimate requires for the metric of $N$ to be at least $C^{2}$. In the alternative proof we are providing, in order to apply lemma 5.1, we only require the almost complex structure and the compatible metric to be $C^{1}$ which corresponds to a weakening of the assumption of magnitude 1 in the derivative.

\subsection{Quantification for harmonic maps on a degenerating surfaces, a coho- moligical condition.}

The aim of this section is to shed a new light on the quantization for harmonic maps on a degenerating surfaces, which has been fully described by M.Zhu in [Zhu.

The main result in the present subsection is the following results which connects energy quantization for harmonic maps into spheres with a cohomological condition.

Theorem 5.2. Let $\left(\Sigma, h_{n}\right)$ be a sequence of closed Riemann surfaces equipped with their constant scalar curvature metric with volume 1 . Let $u_{n}$ be a sequence of harmonic maps from $\left(\Sigma, h_{n}\right)$ into the unit sphere $S^{m-1}$ of the euclidian space $\mathbb{R}^{m}$. Assume

$$
\limsup _{n \rightarrow+\infty} E\left(u_{n}\right)<+\infty
$$


and assume that the following closed forms

$$
\forall i, j=1 \cdots m \quad \star\left(u_{n}^{i} d u_{n}^{j}-u_{n}^{j} d u_{n}^{i}\right)
$$

are all exact. Then the energy quantization holds : modulo extraction of a subsequence, on each component of the limiting thick part, $u_{n}$ converges strongly, away from the punctures, to some limiting harmonic map $u$ and there exists finitely many bubbles, holomorphic maps $\left(\omega^{i}\right)_{i=1 \ldots l}$ from $S^{2}$ into $(N, J)$, - forming possibly both on the thick and the thin parts - such that, modulo extraction of a subsequence

$$
\lim _{n \rightarrow+\infty} E\left(u_{n}\right)=E(u)+\sum_{i=1}^{l} E\left(\omega^{i}\right)
$$

\section{Proof of theorem 5.2:}

In fact, assuming that our sequence of harmonic maps $u_{n}$ get valued into a sphere $S^{m}$, the equation simply write

$$
\Delta u_{n}^{i}=\left(u_{n}^{i} \nabla\left(u_{n}\right)_{j}-\left(u_{n}\right)_{j} \nabla u_{n}^{i}\right) \nabla u_{n}^{j} .
$$

But div $\left(u_{n}^{i} \nabla\left(u_{n}\right)_{j}-\left(u_{n}\right)_{j} \nabla u_{n}^{i}\right)=0=d\left(* u_{n} \wedge d u_{n}\right)$. Hence assuming that the closed $\wedge^{2} \mathbb{R}^{m}$ valued 1-form $\star\left(u_{n} \wedge d u_{n}\right)$ is exact, there exists $b_{n} \in W^{1,2}$ such that

$$
\star\left(u_{n} \wedge d u_{n}\right)=d b_{n},
$$

and

$$
\left\|b_{n}\right\|_{W^{1,2}}=O\left(\left\|u_{n}\right\|_{W^{1,2}}\right)
$$

Then we have

$$
\operatorname{div}\left(\nabla u_{n}-\nabla^{\perp} b_{n} u_{n}\right)=0 .
$$

If we are on a neck region such as $B_{1} \backslash D\left(0, \varepsilon_{n}\right)$, it can be integrated as

$$
\nabla u_{n}=\nabla^{\perp} b_{n} u_{n}+\nabla^{\perp} c_{n}+d_{n} \nabla \log (\rho),
$$

where $c_{n} \in W^{1,2}\left(B_{1}\right)$ and $d_{n} \in \mathbb{R}$. Then we try to control the gradient of the logarithmic part, remarking that

$$
\begin{aligned}
\frac{d}{d \rho} \int_{0}^{2 \pi} u_{n} d \theta & =\int_{0}^{2 \pi} \frac{1}{\rho} \frac{\partial b_{n}}{\partial \theta} u_{k} d \theta+2 \pi \frac{d_{n}}{\rho} \\
& =\int_{0}^{2 \pi} \frac{1}{\rho} \frac{\partial b_{n}}{\partial \theta}\left(u_{k}-\bar{u}_{n}^{\rho}\right) d \theta+2 \pi \frac{d_{n}}{\rho}
\end{aligned}
$$

where $\bar{u}_{n}^{\rho}$ is the mean value of $u_{n}$ over $\partial B_{\rho}$. Integrating the previous identity from $\varepsilon_{n}$ to an arbitrary $\rho$ gives

$$
2 \pi\left(\bar{u}_{n}^{\rho}-\bar{u}_{n}^{\varepsilon_{n}}\right)=\int_{\varepsilon_{n}}^{\rho} \int_{0}^{\pi} \frac{1}{t} \frac{\partial b_{n}}{\partial \theta}\left(u_{k}-\bar{u}_{n}^{t}\right) d \theta d t+2 \pi \log \left(\frac{\rho}{\varepsilon_{n}}\right) .
$$

And, thanks to Poincaré's inequality, we get

$$
\left|\int_{\varepsilon_{n}}^{\rho} \int_{0}^{\pi} \frac{1}{t} \frac{\partial b_{n}}{\partial \theta}\left(u_{k}-\bar{u}_{n}^{t}\right) d \theta d t\right| \leq\left\|\nabla b_{n}\right\|_{2}\left\|\nabla u_{n}\right\|_{2}
$$


Then, combining (94) and (95), we finally obtain that

$$
d_{n}=O\left(\frac{1}{\log \left(\frac{1}{\varepsilon_{n}}\right)}\right)
$$

Which implies, as in the proof of lemma 5.1, that the $L^{2,1}$-norm of $d_{n} \nabla \log (\rho)$ in $B_{1} \backslash D\left(0, \varepsilon_{n}\right)$ is uniformly bounded. By the mean of lemma 5.1 and thanks to (93), we see that he $L^{2,1}$-norm of $\nabla\left(u_{n}-d_{n} \log (\rho)\right)$ is also uniformly bounded and these two uniform bounds imply the uniform $L^{2,1}$ bound of $\nabla u_{n}$ in neck regions. Combining the uniform $L^{2,1}$ bound of $\nabla u_{n}$ in neck regions together with the lemma 3.1 gives the desired energy quantization (92) and theorem 5.2 is proved.

More generally we can raise the following question : Considering a sequence of harmonic maps from a degenerating surface to a general target manifolds, is there is a simple cohomological condition similar as the one in theorem 5.2 ensuring the quantization of the energy in collar region?

\subsection{Energy Quantization for Willmore Surfaces.}

Finally we would like to recall a last application of lemma 5.1 that has been used in a recent work by Y.Bernard and T.Riviere in $[\mathrm{BR}$ for proving Energy Quantization for sequences of Willmore surfaces with uniformly bounded energy and non-degenrating conformal classes. The problem can be described as follows : for a sufficiently smooth immersion $u: \Sigma \rightarrow \mathbb{R}^{m}$, where $\Sigma$ is a closed two dimensional Riemannian surface, we can define its mean curvature vector $\vec{H}$ and we consider the following functional

$$
W(u)=\int_{\Sigma}|\vec{H}|^{2} u^{*}(d y)
$$

where $u^{*}(d y)$ denotes the metric induced on $\Sigma$ by the immersion $u$. This functional is called, the Willmore functional and is known to be conformally invariant (see Ri6]). Critical points to the functional $W$ are called Willmore immersions or Willmore surfaces. Hence as for harmonic maps or pseudo holomorphic curves the question of the quantization of sequences of Willmore surfaces arise naturally. The second author has developed appropriate tools to study weak critical points to $W$ in [Ri4] and [Ri5] and proved the epsilon-regularity for these weak critical points. Using in particular lemma 5.1 the following energy quantization has been established

Theorem 5.3. $[B R]$ Let $u_{n}$ be a sequence of Willmore immersions of a closed surface $\Sigma$. Assume that

$$
\limsup _{n \rightarrow+\infty} W\left(u_{n}\right)<+\infty
$$

that the conformal class of $u_{n}^{*}\left(\xi_{\mathbb{R}^{m}}\right)$ remains within a compact subdomain of the moduli space of $\Sigma$. Then, modulo extraction of a subsequence, the following energy identity holds

$$
\lim _{n \rightarrow+\infty} W\left(u_{n}\right)=W\left(u_{\infty}\right)+\sum_{l=1}^{L} W\left(\omega_{l}\right)+\sum_{k=1}^{K}\left(W\left(\Omega_{k}\right)-4 \pi \theta_{k}\right)
$$

where $u_{\infty}$ is a possibly branched smooth Willmore immersion of $\Sigma$. The maps $\omega_{l}$ and $\Omega_{k}$ are smooth, possibly branched, Willmore immersions of $S^{2}$ and $\theta_{k}$ is the integer density of the current $\left(\Omega_{k}\right)_{*}\left(S^{2}\right)$ at some point $p_{k} \in \Omega_{k}\left(S^{2}\right)$, namely

$$
\theta_{k}=\lim _{\rho \rightarrow 0} \frac{\mathcal{H}^{2}\left(B_{r}(p) \cap \Omega_{k}\left(S^{2}\right)\right)}{\pi \rho^{2}} .
$$




\section{A Lorentz Estimates on Harmonic Functions.}

Here we prove two lemmas on harmonic which insure that we can control the $L^{2,1}$-norm by the $L^{2}$ on a smaller domain up to some appropriate boundary condition.

Lemma A.1. Let $0<\varepsilon<\frac{1}{2}$ and $f: B_{1} \backslash B_{\varepsilon} \rightarrow \mathbb{R}$ an harmonic function which satisfies

$$
\begin{aligned}
& f=0 \text { on } \partial B_{1}, \\
& \int_{\partial B_{\varepsilon}} f d \sigma=0 .
\end{aligned}
$$

Then, for all $\lambda>1$, there exists positive a constant $C(\lambda)$ independent of $\varepsilon$ and $f$ such that

$$
\|\nabla f\|_{L^{2,1}\left(B_{1} \backslash B_{\lambda \varepsilon}\right)} \leq C(\lambda)\|\nabla f\|_{2} .
$$

Proof of lemma A.1:

We start by decomposing $f$ as a Fourier series, which gives

$$
f(\rho, \theta)=c_{0}+d_{0} \ln (\rho)+\sum_{n \in \mathbb{Z}^{*}}\left(c_{n} \rho^{n}+d_{n} \rho^{-n}\right) e^{i n \theta} .
$$

Hence, using (96), we easily proved that $c_{0}=d_{0}=c_{n}+d_{n}=0$, then we get

$$
f(\rho, \theta)=\sum_{n \in \mathbb{Z}^{*}} c_{n}\left(\rho^{n}-\rho^{-n}\right) e^{i n \theta}
$$

Then we estimate the gradient as follows

$$
|\nabla f(\rho, \theta)| \leq 2 \sum_{n \in \mathbb{Z}^{*}}\left|n c_{n}\right|\left(\rho^{n-1}+\rho^{-n-1}\right) .
$$

Then, we estimate the $L^{2,1}$-norm of the $f_{m}(z)=|z|^{m}$ on $B_{1} \backslash B_{\lambda \varepsilon}$, for $m \in \mathbb{Z} \backslash\{-1\}$ and $\left.\left.\lambda \in\right] 1,2\right]$, which gives

$$
\begin{aligned}
& \left\|f_{m}\right\|_{L^{2,1}\left(B_{1} \backslash B_{\lambda \varepsilon}\right)} \leq \sqrt{\pi} \int_{0}^{(\lambda \varepsilon)^{m}} t^{\frac{1}{m}} d t \leq 2 \sqrt{\pi}(\lambda \varepsilon)^{m+1} \text { for } m<-1 \\
& \text { and } \\
& \left\|f_{m}\right\|_{L^{2,1}\left(B_{1} \backslash B_{\lambda \varepsilon}\right)} \leq \sqrt{\pi} \text { for } m \geq 0 .
\end{aligned}
$$

Here we use the following characterization (9). Hence we get

$$
\|\nabla f\|_{L^{2,1}\left(B_{1} \backslash B_{\lambda \varepsilon}\right)} \leq 4 \sqrt{\pi}\left(\sum_{n>0}\left|n c_{n}\right|\left((\lambda \varepsilon)^{-n}+1\right)+\sum_{n<0}\left|n c_{n}\right|\left((\lambda \varepsilon)^{n}+1\right)\right) .
$$

Hence, thanks to the Cauchy-Scharwz and the fact that $\lambda>1$, we get

$$
\|\nabla f\|_{L^{2,1}\left(B_{1} \backslash B_{\lambda \varepsilon}\right)} \leq 8 \sqrt{\pi}\left(\sum_{n \neq 0}|n| \lambda^{-2|n|}\right)\left(\sum_{n \neq 0}|n|\left|c_{n}\right|^{2} \varepsilon^{-2|n|}\right)^{\frac{1}{2}} .
$$


Finally we compute the $L^{2}$-norm of $\nabla f$

$$
\|\nabla f\|_{2}=\left(2 \pi \int_{\varepsilon}^{1} \sum_{n \neq 0}\left|n c_{n}\right|^{2}\left(\rho^{2 n-2}+\rho^{-2 n-2}\right) \rho d \rho\right)^{\frac{1}{2}} \geq \sqrt{\frac{\pi}{2}}\left(\sum_{n \neq 0}|n|\left|c_{n}\right|^{2} \varepsilon^{-2|n|}\right)^{\frac{1}{2}}
$$

which achieves the proof of lemma A.1.

Lemma A.2. let $0<\varepsilon<\frac{1}{4}$ and $f: B_{1} \backslash B_{\varepsilon} \rightarrow \mathbb{R}$ an harmonic function which satisfies

$$
\begin{aligned}
& \int_{\partial B_{\varepsilon}} f d \sigma=0, \\
& \left|\int_{\partial B_{1}} f d \sigma\right| \leq K,
\end{aligned}
$$

where $K$ is a constant independent of $\varepsilon$. Then, for all $0<\lambda<1$ there exists positive constant $C(\lambda)$ independent of $\varepsilon$ and $f$ such that

$$
\left.\|\nabla f\|_{L^{2,1}\left(B_{\lambda} \backslash B_{\lambda}-1_{\varepsilon}\right.}\right) \leq C(\lambda)\left(\|\nabla f\|_{2}+1\right) .
$$

Proof of lemma A.2:

We start by decomposing $f$ as a Fourier series, which gives

$$
f(\rho, \theta)=c_{0}+d_{0} \ln (\rho)+\sum_{n \in \mathbb{Z}^{*}}\left(c_{n} \rho^{n}+d_{n} \rho^{-n}\right) e^{i n \theta} .
$$

Hence, using (98), we easily proved that $c_{0}+d_{0} \ln (\varepsilon)=0$ and $\left|c_{0}\right|=O(1)$. Hence

$$
d_{0}=O\left(\frac{-1}{\ln (\varepsilon)}\right) \text {. }
$$

Then we estimate the gradient as follows

$$
|\nabla f(\rho, \theta)| \leq\left|d_{0}\right| \frac{1}{\rho}+\sum_{n \in \mathbb{Z}^{*}}\left|n c_{n}\right| \rho^{n-1}+\left|n d_{n}\right| \rho^{-n-1} .
$$

Then, we estimate the $L^{2,1}$-norm of the $f_{m}(z)=|z|^{m}$ on $B_{\lambda} \backslash B_{\lambda^{-1} \varepsilon}$, for $m \in \mathbb{Z} \backslash\{-1\}$ and $0<\lambda<1$, which gives

$$
\begin{aligned}
& \left\|f_{m}\right\|_{2,1} \leq \sqrt{\pi} \int_{0}^{\left(\lambda^{-1} \varepsilon\right)^{m}} t^{\frac{1}{m}} d t \leq 2 \sqrt{\pi}\left(\lambda^{-1} \varepsilon\right)^{m+1} \text { for } m<-1 \\
& \left\|f_{m}\right\|_{2,1} \leq \sqrt{\pi} \lambda^{m} \text { for } m \geq 0 \\
& \text { and } \\
& \left\|f_{-1}\right\|_{2,1}=O(-\log (\varepsilon)) .
\end{aligned}
$$

Here we use the following characterization (9). Thanks to (99) and (100), we get

$$
\begin{aligned}
\left.\|\nabla f\|_{L^{2,1}\left(B_{\lambda} \backslash B_{\lambda-1} \varepsilon\right.}\right) & \leq 2 \sqrt{\pi}\left(\sum_{n>0}\left(\left|n c_{n}\right| \lambda^{n}+\left|n d_{n}\right|\left(\lambda^{-1} \varepsilon\right)^{-n}\right)+\sum_{n<0}\left(\left|n c_{n}\right|\left(\lambda^{-1} \varepsilon\right)^{n}+\left|n d_{n}\right| \lambda^{-n}\right)\right) \\
& +O(1) .
\end{aligned}
$$


Hence, thanks to the Cauchy-Scharwz and the fact that $0<\lambda<1$, we get

$$
\begin{aligned}
\|\nabla f\|_{L^{2,1}\left(B_{\lambda} \backslash B_{\lambda^{-1} \varepsilon}\right)} & \leq 4 \sqrt{\pi}\left(\sum_{n \neq 0}|n| \lambda^{2|n|}\right)\left(\sum_{n<0}|n|\left(\left|c_{n}\right|^{2}+\left|d_{-n}\right|^{2}\right) \varepsilon^{-2|n|}+\sum_{n>0}|n|\left(\left|c_{n}\right|^{2}+\left|d_{-n}\right|^{2}\right) 2^{-n}\right)^{\frac{1}{2}} \\
& +O(1) .
\end{aligned}
$$

Finally we compute the $L^{2}$-norm of $\nabla f$

$$
\begin{aligned}
\|\nabla f\|_{2} & =\left|d_{0}\right|\left(\int_{\varepsilon}^{1} \frac{1}{\rho} d \rho\right)^{\frac{1}{2}}+\left(2 \pi \int_{\varepsilon}^{1} \sum_{n \neq 0}\left(\left|n c_{n}\right|^{2} \rho^{2 n-2}+\left|n d_{n}\right|^{2} \rho^{-2 n-2}\right) \rho d \rho\right)^{\frac{1}{2}} \\
& \geq \sqrt{\frac{\pi}{2}}\left(\sum_{n<0}|n|\left(\left|c_{n}\right|^{2}+\left|d_{-n}\right|^{2}\right) \varepsilon^{-2|n|}+\sum_{n>0}|n|\left(\left|c_{n}\right|^{2}+\left|d_{-n}\right|^{2}\right) 2^{-n}\right)^{\frac{1}{2}}
\end{aligned}
$$

which achieves the proof of lemma A.2.

\section{References}

[Ad] Adams, David R. "A note on Riesz potentials." Duke Math. J. 42 (1975), no. 4, 765-778.

[AdFo] Adams, Robert A. and Fournier, John J. F., "Sobolev Spaces" Elsevier Academic Press, Amsterdam, 2003.

[BR] Bernard, Yann; Rivière, Tristan "Energy Quantization for Willmore Surfaces and Applications" preprint 2011.

[Bet] Bethuel, Fabrice "Un rsultat de rgularité pour les solutions de l'quation de surfaces courbure moyenne prescrite." (French) [A regularity result for solutions to the equation of surfaces of prescribed mean curvature] C. R. Acad. Sci. Paris Sr. I Math. 314 (1992), no. 13, 1003-1007.

[BeRe] Bethuel, Fabrice and Rey, Olivier "Multiple solutions to the Plateau problem for nonconstant mean curvature" Duke Math. J. 73, (1994) 593-646.

[CaMu] Caldiroli, Paolo and Musina, Roberta, "The Dirichlet problem for $H$-systems with small boundary data: blowup phenomena and nonexistence results" Arch. Ration. Mech. Anal. 181, (2006) 1-42.

[CLMS] R.Coifman, P.-L.Lions, Y.Meyer and S.Semmes "Compensated Compactness and Hardy Spaces" J. Math. Pure Appl. 72 (1993), 247-286.

[DaL] Da Lio Francesca, "Energy Quantization for half-harmonic Maps into Spheres" in preparation 2011.

[DiT] W. Ding and G. Tian "Energy identity for a class of approximate harmonic maps from surfaces" Comm. Anal. Geom. 3 (1995), no.3-4, 543-554.

[Ge] Ge, Yuxin "Estimations of the best constant involving the $L^{2}$ norm in Wente's inequality and compact $H$-Surfaces in Euclidian space." C.O.C.V., 3, (1998), 263-300.

[Gra] L. Grafakos Classical Fourier Analysis. Graduate Texts in Mathematics 249, Springer (2009). 
[Gr] Gromov, M., "Pseudo holomorphic curves in symplectic manifolds", Invent. Math. 82, (1985) 307347.

[HaLi] Han, Qing and Lin, Fanghua, "Elliptic partial differential equations" Courant Lecture Notes in Mathematics, 1, New York University Courant Institute of Mathematical Sciences, (1997).

[He] F.Hélein "Harmonic maps, conservation laws and moving frames" Cambridge Tracts in Math. 150, Cambridge Univerity Press, 2002.

[Hub] Hubbard, John Hamal, "Teichmüller theory and applications to geometry, topology, and dynamics." Vol. 1 Matrix Editions, Ithaca, NY (2006).

[Hum] C. Hummel: Gromovs compactness theorem for pseudo-holomorphic curves. Progress in Mathematics 151, Birkhauser Verlag, Basel (1997)

[Jo] J. Jost, "Two-dimensional geometric variational problems", Wiley, (1991).

[LaRi] Laurain, Paul and Rivière, Tristan "Quantification for four order elliptic sytem" in preparation 2011.

[LR1] F.H. Lin and T. Rivière "A Quantization property for static Ginzburg-Landau Vortices" Comm. Pure App. Math.,54 , (2001), 206-228.

[LR2] F.H.Lin and T.Rivière "Energy Quantization for Harmonic Maps" Duke Math. Journal, 111 (2002) 177-193.

[LiWa] F.H. Lin and C.Y. Wang "Energy identity of harmonic map flows from surfaces at finite singular time", Cal. Var. and P.D.E. 6 (1998), no 4, 369-380.

[McDS] McDuff, Dusa and Salamon, Dietmar, "J-holomorphic curves and symplectic topology" American Mathematical Society Colloquium Publications 52, American Mathematical Society, 2004.

[Pa] T.Parker, "Bubble tree convergence for harmonic maps", J. Diff. Geom. 44 (1996), 545-633.

[PW] Parker, Thomas H. and Wolfson, Jon G. "Pseudo-holomorphic maps and bubble trees" J. Geom. Anal. 3, (1993) 63-98.

[Ri1] T. Rivière "Interpolation spaces and energy quantization for Yang-Mills fields" Comm. Anal. Geom. 8 (2002).

[Ri2] Rivière, Tristan "Bubbling and regularity issues in geometric non-linear analysis" Proceedings of the International Congress of Mathematicians, Vol. III (Beijing, 2002), 197208, Higher Ed. Press, Beijing, 2002.

[Ri3] Rivière, Tristan "Conservation laws for conformally invariant variational problems." Invent. Math. 168 (2007), no. 1, 1-22.

[Ri4] Rivière, Tristan "Analysis aspects of Willmore surfaces", Inventiones Math., 174 (2008), no.1, 1-45.

[Ri5] Rivière, Tristan "Variational Principles for immersed Surfaces with $L^{2}$-bounded Second Fundamental Form." arXiv:1007.2997 july 2010.

[Ri6] Rivière, Tristan "Conformally Invariant 2-dimensional Variational Problems" Cours joint de l'Institut Henri Poincaré - Paris XII Creteil, Novembre 2010.

[SaU] J. Sacks and K. Uhlenbeck, The existence of minimal immersions of 2-spheres, Ann. of Math. 113 (1981), 1-24. 
[ShTo] Ben Sharp and Peter Topping "Decay estimates for Rivière's equation, with applications to regularity and compactness", http://arxiv.org/abs/1102.0713.

[Si] Sikorav "Holomorphic curves in symplectic geometry" Ed. Audin, M. and Lafontaine, J., Some properties of holomorphic curves in almost complex manifolds, Birkhäuser 1994.

[Ste] E.M. Stein, Singular integrals and differentiability properties of functions. Princeton Mathematical Series, No. 30 Princeton University Press, Princeton, N.J. 1970.

[StWe] E.M. Stein \& G. Weiss, Introduction to Fourier analysis on Euclidean spaces. Princeton Mathematical Series, No. 32. Princeton University Press, Princeton, N.J., 1971.

[St] M.Struwe "On the evolution of harmonic mappings of Riemannian surfaces" Comm. Math. Helvetici 60 (1985) 558-581.

[Tar] Tartar, Luc "Remarks on oscillations and Stokes' equation. Macroscopic modelling of turbulent flows" (Nice, 1984), 24-31, Lecture Notes in Phys., 230, Springer, Berlin, 1985

[To] Topping, Peter "The optimal constant in Wente's $L^{\infty}$ estimate", Comm. Math. Helv. 72, (1997), $316-328$

[We] Wente, Henry C. "An existence theorem for surfaces of constant mean curvature", J. Math. Anal. Appl. 26 (1969), 318-344.

[Zhu] Zhu, Miaomiao Harmonic maps from degenerating Riemann surfaces. Math. Z. 264 (2010), no. 1, 6385 . 